\title{
Racial and Ethnic Disparities in Years of Potential Life Lost Attributable to COVID-19 in the United States: An Analysis of 45 States and the District of Columbia
}

\author{
Jay J. Xu ${ }^{1, *,+(\mathbb{D})}$, Jarvis T. Chen ${ }^{2} \mathbb{D}$, Thomas R. Belin ${ }^{1,3} \mathbb{D}$, Ronald S. Brookmeyer ${ }^{1}\left(\mathbb{D}\right.$ and Marc A. Suchard ${ }^{1,4,5}$ \\ and Christina M. Ramirez ${ }^{1}$ (D)
}

Citation: Xu, J.J.; Chen, J.T.; Belin, T.R.; Brookmeyer, R.S.; Suchard, M.A. and Ramirez, C.M. Racial and Ethnic Disparities in Years of Potential Life Lost Attributable to COVID-19 in the United States: An Analysis of 45 States and the District of Columbia. Int. J. Environ. Res. Public Health 2021, 18, 2921. https://doi.org/10.3390/ ijerph18062921

Academic Editor: Jayajit Chakraborty

Received: 28 January 2021

Accepted: 8 March 2021

Published: 12 March 2021

Publisher's Note: MDPI stays neutral with regard to jurisdictional claims in published maps and institutional affiliations.

Copyright: (c) 2021 by the authors. Licensee MDPI, Basel, Switzerland. This article is an open access article distributed under the terms and conditions of the Creative Commons Attribution (CC BY) license (https:/ / creativecommons.org/licenses/by/ $4.0 /)$.
1 Department of Biostatistics, Jonathan and Karin Fielding School of Public Health, University of California, Los Angeles, CA 90095, USA; tbelin@mednet.ucla.edu (T.R.B.); rbrookmeyer@ucla.edu (R.S.B.); msuchard@ucla.edu (M.A.S.); cr@ucla.edu (C.M.R.)

2 Department of Social and Behavioral Sciences, Harvard T.H. Chan School Of Public Health, Harvard University, Cambridge, MA 02115, USA; jarvis@hsph.harvard.edu (J.T.C.)

3 Department of Psychiatry and Biobehavioral Sciences, David Geffen School of Medicine, University of California, Los Angeles, CA 90095, USA

4 Department of Human Genetics, David Geffen School of Medicine, University of California, Los Angeles, CA 90095, USA

5 Department of Computational Medicine, David Geffen School of Medicine, University of California, Los Angeles, CA 90095, USA

* Correspondence: jayxu33@ucla.edu

+ Current address: 650 Charles E. Young Drive South, 51-254 CHS, Los Angeles, CA 90095, USA.

Abstract: The coronavirus disease 2019 (COVID-19) epidemic in the United States has disproportionately impacted communities of color across the country. Focusing on COVID-19-attributable mortality, we expand upon a national comparative analysis of years of potential life lost (YPLL) attributable to COVID-19 by race/ethnicity (Bassett et al., 2020), estimating percentages of total YPLL for non-Hispanic Whites, non-Hispanic Blacks, Hispanics, non-Hispanic Asians, and non-Hispanic American Indian or Alaska Natives, contrasting them with their respective percent population shares, as well as age-adjusted YPLL rate ratios-anchoring comparisons to non-Hispanic Whites-in each of 45 states and the District of Columbia using data from the National Center for Health Statistics as of 30 December 2020. Using a novel Monte Carlo simulation procedure to perform estimation, our results reveal substantial racial/ethnic disparities in COVID-19-attributable YPLL across states, with a prevailing pattern of non-Hispanic Blacks and Hispanics experiencing disproportionately high and non-Hispanic Whites experiencing disproportionately low COVID-19-attributable YPLL. Furthermore, estimated disparities are generally more pronounced when measuring mortality in terms of YPLL compared to death counts, reflecting the greater intensity of the disparities at younger ages. We also find substantial state-to-state variability in the magnitudes of the estimated racial/ethnic disparities, suggesting that they are driven in large part by social determinants of health whose degree of association with race/ethnicity varies by state.

Keywords: communities of color; coronavirus; COVID-19; medical mistrust; Monte Carlo simulation; public health; racial and ethnic disparities; SARS-CoV-2; social determinants of health; years of potential life lost

\section{Introduction}

Severe acute respiratory syndrome coronavirus 2 (SARS-CoV-2) [1], the coronavirus that causes coronavirus disease 2019 (COVID-19), has infected 192 countries and territories across the world [2]. First identified in an outbreak in Wuhan, Hubei province, China in December 2019, COVID-19 rapidly proliferated across the world, with the first confirmed case in the United States (U.S.) identified on 20 January 2020 [3,4]. On 11 March 2020, the World 
Health Organization officially declared the COVID-19 outbreak a global pandemic [5], and by the end of 2020, the U.S. has had over 20 million confirmed cases of COVID-19 and $346,000+$ deaths [6], both figures the highest among any country in the world. COVID-19 doesn't affect all segments of the population equally, however, with males and older individuals at higher risk of post-infection mortality for example [7,8]. Statistics on COVID-19 outcomes that local and state governmental health agencies in the U.S. publicly released did not initially include racial/ethnic demographic information on confirmed cases or deaths. Public pressure quickly grew for such information to be provided $[9,10]$, with many local and state governmental health agencies quickly following suit. Racial/ethnic data on COVID-19 outcomes have revealed substantial racial/ethnic disparities, with non-Hispanic Blacks, Hispanics, and non-Hispanic American Indian or Alaska Natives experiencing disproportionately high numbers of cases, hospitalizations, and deaths [11]. Racial and ethnic disparities in outcomes associated with COVID-19 in the U.S. have been the subject of widespread concern and societal discourse. Characterizing racial/ethnic disparities in COVID-19 outcomes is an active area of scientific research [12-31], and quantifying racial/ethnic disparities in the COVID-19 mortality burden has been a particular area of focus. For example, the Color of Coronavirus project of the American Public Media Research Lab [32] tracks COVID-19 death counts by race/ethnicity in each state, providing analyses and extended commentary on mortality rates by race/ethnicity and comparisons between the percentage of total deaths and the percent population share by race/ethnicity. Another similar data hub for COVID-19 mortality statistics by race/ethnicity is the COVID Racial Data Tracker of the COVID Tracking Project [33].

Given the substantially greater COVID-19 case fatality rates experienced by individuals in older age groups, COVID-19 death counts and mortality rates are predominantly determined by data from COVID-19 decedents in older age groups. Younger individuals, however, are also susceptible to death from COVID-19, which in principle represent greater unrealized years of life, economic productivity, and broader contributions to society compared to decedents of greater age. An alternative epidemiological measure of mortality that explicitly weights deaths that occur at earlier ages more heavily than deaths that occur at later ages is years of potential life lost (YPLL) [34]. YPLL for an individual fatality $i$ is defined to be the difference between an upper reference age $\mathcal{A}$ and age at death $a_{i}$ if the difference is positive and zero otherwise:

$$
\mathrm{YPLL}_{i}=\max \left\{\mathcal{A}-a_{i}, 0\right\}
$$

When comparing racial/ethnic subgroups with disparate mortality rates in younger age groups, higher YPLL is expected for those subgroups with comparatively higher mortality rates in the younger age groups, assuming a constant upper reference age is used across racial/ethnic groups. YPLL has been used in diverse contexts to quantify racial/ethnic disparities in premature mortality [35-37]. In the context of COVID-19, Bassett et al. (2020) [38] performed an analysis of COVID-19 deaths in the U.S. as a whole using COVID-19 mortality data from the National Center for Health Statistics as of 22 July 2020, calculating both age-specific mortality rates and YPLL by race/ethnicity. They found substantial racial/ethnic disparities in mortality rates across all age groups, but the magnitudes of the disparities were larger within younger age groups, which was reflected in their calculations of YPLL (with $\mathcal{A}=65$ ). Although non-Hispanic Blacks and Hispanics both comprise a smaller percentage of the U.S. population than non-Hispanic Whites, Bassett et al. found that both non-Hispanic Blacks and Hispanics experienced greater COVID-19-attributable YPLL than non-Hispanic Whites, which was largely due to comparatively higher mortality rates in younger age groups.

Here, we extend the work of Bassett et al. by quantifying racial/ethnic disparities in YPLL attributable to COVID-19 at the state level, which represents a more comprehensive characterization of racial/ethnic disparities in COVID-19-attributable YPLL in the U.S. and allows for the identification of specific states where certain racial/ethnic groups have been particularly hard hit by COVID-19. Specifically, we characterize racial/ethnic 
disparities in COVID-19-attributable YPLL through the estimation of percentages of total YPLL for non-Hispanic Whites, non-Hispanic Blacks, Hispanics, non-Hispanic Asians, and non-Hispanic American Indian and Alaska Natives, contrasting them with their respective percent population shares, in each of 45 states and the District of Columbia (D.C.). Moreover, to ensure comparability of YPLL estimates by race/ethnicity given the differences in the population age distributions between racial/ethnic groups both within and between states, we further characterize racial/ethnic disparities by estimating age-adjusted YPLL rate ratios (RR), anchoring comparisons to non-Hispanic Whites. For comparison, we also calculate racial/ethnic percentages of total COVID-19 deaths and estimate the corresponding age-adjusted mortality RR's to examine potential differences in the characterization of racial/ethnic disparities when measuring mortality in terms of YPLL compared to death counts. Lastly, we use an improved statistical framework for uncertainty quantification of the YPLL-based quantities of interest compared to Bassett et al. adopting a finite-population inferential paradigm and using novel Monte Carlo (MC) simulation techniques for interval estimation.

\section{Materials and Methods}

\subsection{Data}

We examine U.S. national COVID-19 mortality data from the National Center for Health Statistics (NCHS) as of 30 December 2020 - the last data update for calendar year 2020 - summarized as cumulative death counts within age groups stratified by state (as well as D.C. and Puerto Rico) and race/ethnicity [39], which we refer to hereafter as the NCHS Race/Ethnicity Data. The following 8 racial/ethnic groups are used in the NCHS Race/Ethnicity Data: non-Hispanic White (NH White), non-Hispanic Black (NH Black), Hispanic, non-Hispanic Asian (NH Asian), non-Hispanic American Indian or Alaska Native (NH AIAN), non-Hispanic Native Hawaiian or Other Pacific Islander, non-Hispanic Two or More Races, and Unknown. The set of mutually exclusive, collectively exhaustive, and chronologically ordered age groups used are $<1,1-4,5-14,15-24,25-34,35-44,45-54$, 55-64, 65-74, 75-84 and 85+. See File S1 in the Supplementary Materials for the NCHS Race/Ethnicity Data as of 30 December 2020, which consists of 301,679 total deaths. Because there is a lag in time between the actual date of death and when the death certificate is completed, submitted to the NCHS, and processed, this number does not reflect the actual number of deaths in the U.S. as of 30 December 2020, which was reported to be 342,577 by the New York Times [6]. Some states' data reported to the NCHS have been documented as severely delayed [40-42], especially North Carolina, which is one of a few states that, at the time of writing, doesn't use an electronic death registration system [43]. Our analysis of the NCHS Race/Ethnicity Data implicitly assumes that there is no systematic bias in the speed with which death certificates are reported by states to the NCHS with respect to race/ethnicity or age at death.

Death counts between 1 and 9 within individual age groups are suppressed in the NCHS Race/Ethnicity Data due to confidentiality regulations. A total of 1160 age groups across the 50 states and D.C. and $8 \mathrm{racial} / \mathrm{ethnic}$ groups have suppressed death counts. Although the NCHS Race/Ethnicity Data do not explicitly provide the total number of actual deaths in each jurisdiction, the NCHS provides a separate but synchronously updated publicly available dataset of COVID-19 deaths summarized as cumulative death counts within the same age groups as the NCHS Race/Ethnicity Data stratified by state and-instead of race/ethnicity—sex, which we refer to hereafter as the NCHS Sex Data [44], that does explicitly provide the total number of actual deaths in each state; see File S2 in the Supplementary Materials for the NCHS Sex Data as of 30 December 2020. Hence, for each state, we can ascertain the total number of deaths that are within the union of age groups with suppressed death counts, each of which contains between 1 and 9 deaths. The NCHS Race/Ethnicity Data also provides non-suppressed death counts within these same age groups stratified by race/ethnicity for the U.S. overall. 
To standardize estimates by age, we make use of 2019 estimates of the U.S. population age distribution stratified by state (and D.C.), race, and ethnicity from CDC WONDER [45], defined over integer ages from 0 to 84 and a catch-all $85+$ age group. Percent population shares by race/ethnicity both nationally and in each state (and D.C.) are calculated from the 2019 CDC WONDER data. See File S3 in the Supplementary Materials for the 2019 CDC WONDER data.

\subsection{Estimation Procedure for YPLL-Based Estimands from Administratively Interval Censored Ages at Death}

As previously described in Section 1, we characterize racial/ethnic disparities in COVID-19-attributable YPLL through the estimation of percentages of total YPLL by race/ethnicity, contrasting them with their respective percent population shares, as well as age-adjusted YPLL RR's, anchoring comparisons to NH Whites. To provide additional perspective on the sheer magnitude of COVID-19-attributable YPLL by race/ethnicity, we also perform estimation of total YPLL and age-adjusted YPLL rates by race/ethnicity. To quantify the uncertainty of their estimates of total YPLL, age-adjusted YPLL rates, and age-adjusted YPLL RR's, Bassett et al. adopted a super-population inferential perspective, where observed COVID-19 deaths were viewed as a single realization of a hypothetical probability distribution, and frequentist statistical theory was used for interval estimation [46]. In contrast, we view the finite-population perspective that the observed COVID-19 deaths simply constitute the population of interest to be more scientifically appropriate, and as such, estimation uncertainty pertaining to the YPLL-based estimands of interest can be attributed to three sources: (a) administrative interval censoring of ages at death, (b) suppression of low death counts within individual age intervals, and (c) unknown race/ethnicity for a subset of deaths.

Focusing first on the issue of administrative interval censoring of ages at death and assuming no suppressed death counts or deaths of unknown race/ethnicity for purposes of illustration, the unknown exact ages at death for each individual precludes exact calculation of individual YPLL values. For the purpose of calculating aggregate YPLL, the standard approach in such settings is to operationally impute each individual's age at death with its interval midpoint, a method that implicitly assumes uniformly distributed ages at death within individual age intervals [47]. However, applied epidemiological studies employing this "midpoint method" typically do not quantify the uncertainty associated with YPLLbased estimates as a result of the administrative interval censoring of ages at death [48-51]. $\mathrm{Xu}$ et al. (2021) [52] proposed a MC simulation procedure to quantify the uncertainty associated with YPLL-based estimates obtained from mortality data summarized as death counts within age intervals. We refer the reader to their paper for the details of the MC simulation procedure, but to summarize it briefly, the procedure comprises independently simulating ages at death for each individual from continuous uniform distributions defined over their respective age intervals at each MC iteration, calculating a point estimate of the estimand of interest from the corresponding simulated YPLL values. The overall point estimate is defined to be the mean of the collection of MC point estimates, and the lower and upper endpoints of a $(1-\alpha) \times 100 \%$ interval estimate (which can be conceptualized as a "range interval" per Bobashev and Morris (2010) [53]) are defined to be the $\frac{\alpha}{2}$ and $1-\frac{\alpha}{2}$ quantiles of the collection of MC point estimates, respectively.

\subsection{Procedure Modification to Account for Suppression of Low Death Counts}

The second source of estimation uncertainty, suppression of low death counts within individual age intervals, arises out of privacy and confidentiality concerns associated with publishing small death counts within individual age intervals, which can risk revealing personally identifiable information for individual COVID-19 deaths. We introduce a modification of the Xu et al. MC simulation procedure described in Section 2.2 to account for this additional source of estimation uncertainty while still assuming no deaths of unknown race/ethnicity for purposes of illustration. 
For each state, we know the total number of deaths contained in the union of intervals with suppressed death counts, each of which must be an integer between 1 and 9. As such, we can exhaustively enumerate all possible death count combinations across the intervals with suppressed death counts. Each death count combination corresponding to the intervals with suppressed death counts juxtaposed with the intervals containing non-suppressed death counts constitutes one possible "mortality dataset" of death counts within age intervals. We modify the Xu et al. MC simulation procedure by independently simulating ages at death for each individual for each mortality dataset at each MC iteration. Then, a point estimate of the estimand of interest is calculated for each mortality dataset from the corresponding simulated YPLL values, from which we store only the minimum and maximum point estimate across the mortality datasets at each $\mathrm{MC}$ iteration. A conservative $(1-\alpha) \times 100 \%$ interval estimate of the estimand of interest is then constructed from the $\frac{\alpha}{2}$ quantile of the collection of minimum MC point estimates and the $1-\frac{\alpha}{2}$ quantile of the collection of maximum MC point estimates. We describe the interval estimate as "conservative" as a result of our estimation strategy of considering all possible mortality datasets and using the extrema of the subsequent MC point estimates to construct the interval estimate; a $(1-\alpha) \times 100 \%$ interval estimate of the estimand of interest obtained from the standard $\mathrm{Xu}$ et al. MC simulation procedure had the suppressed death counts been known (and assuming no deaths with unknown race/ethnicity) would be completely contained in the corresponding conservative $(1-\alpha) \times 100 \%$ interval estimate.

\subsection{Procedure Modification to Further Account for a Subset of Deaths with Unknown Race/Ethnicty}

The third source of estimation uncertainty, unknown race/ethnicity for a subset of deaths, can be accommodated through an additional modification of the Xu et al. MC simulation procedure. The key idea is to separate the tasks of obtaining a maximum and minimum point estimate at each MC iteration. Specifically, when the estimand of interest primarily concerns racial/ethnic group $r$ (i.e., total YPLL for racial/ethnic group $r$, percentage of total YPLL for racial/ethnic group $r$, age-adjusted YPLL rate for racial/ethnic group $r$, and age-adjusted $r$-to-NH White YPLL RR), we assume all deaths among the Unknown racial/ethnic group are members of racial/ethnic group $r$ and appropriately combine death counts within their age intervals before exhaustively enumerating all possible mortality datasets, simulating ages at death for each individual for each mortality dataset, calculating a point estimate from the corresponding simulated YPLL values for each mortality datset, and storing the maximum point estimate across mortality datasets at each MC iteration. The task of obtaining a minimum point estimate at each MC iteration is pursued analogously but separately, where the key distinction is that we do not combine the Unknown racial/ethnic group and racial/ethnic group $r$. Therefore, a separate set of mortality datasets is considered for each task of maximization and minimization.

\subsection{Computational Savings by Omitting Unnecessary Mortality Datasets}

The total number of mortality dataset scenarios can be enormous, potentially making it computationally infeasible to simulate ages at death for all mortality datasets. However, substantial computational savings can be achieved by (a) combining racial/ethnic groups not included in the definition of the estimand of interest and (b) identifying mortality datasets that we do not need to simulate ages at death from because they would yield a maximum or minimum MC point estimate with probability 0 . We describe an example of such combinatorial reductions when the estimand of interest is the percentage of total YPLL for racial/ethnic group $r$.

For the task of obtaining a maximum point estimate at each $\mathrm{MC}$ iteration, we combine the Unknown racial/ethnic group with racial/ethnic group $r$, combine the remaining racial/ethnic groups into a single non- $r$ racial/ethnic group, which we denote $\bar{r}$, and enumerate all possible mortality datasets. In this case, only one mortality dataset needs to be considered, namely, the one that contains the maximum possible number of deaths in the lowest age intervals corresponding to suppressed death counts for racial/ethnic 
group $r$. The remaining mortality datasets can be omitted because they would yield a maximum MC point estimate with probability 0. For the task of obtaining a minimum point estimate at each MC iteration, we combine all of the non- $r$ racial/ethnic groups (including the Unknown racial/ethnic group) into a single non-r racial/ethnic group $\bar{r}$ and enumerate all possible mortality datasets. Here, the only mortality dataset that needs to be considered is the one that contains the maximum possible number of deaths in the lowest age intervals corresponding to suppressed death counts for the combined non- $r$ racial/ethnic group $\bar{r}$ and, if necessary, the maximum possible number of deaths in the highest age intervals corresponding to suppressed death counts for racial/ethnic group $r$. The remaining mortality datasets can be omitted because they would yield a minimum MC point estimate with probability 0.

For estimation of the age-adjusted YPLL rates and RR's, identifying mortality datasets that we don't need to simulate ages at death from is a more challenging task, but it is still possible. Inequality conditions can be established computationally to identify meaningful numbers of mortality datasets that can be omitted in our analysis.

\subsection{Complete Monte Carlo Simulation Procedure for Estimation of YPLL-Based Estimands}

Here, we comprehensively summarize the complete modified $\mathrm{Xu}$ et al. MC simulation procedure for estimation of the YPLL-based estimands of interest that quantifies the uncertainty associated with the administrative interval censoring of ages at death, suppression of low death counts within individual age intervals, and unknown race/ethnicity for a subset of deaths. We limit the analysis presented here to jurisdictions in the continental U.S. with at least 500 total deaths in the NCHS Sex Data, a condition satisfied by 45 states as well as D.C., which for brevity we characterize as a "state" in summaries of the results of our analysis. The 5 states omitted in our analysis are Alaska, Hawaii, Maine, Vermont, and Wyoming. The criterion of at least 500 deaths was used as the benchmark for inclusion in the analysis because for states with a small number of COVID-19 deaths, comparisons between racial/ethnic groups, each having an even smaller number of death counts which are potentially partially suppressed, would yield findings with limited practical value coupled with extremely large interval estimates. For each examined state $s$, the procedure can be comprehensively summarized as follows.

1. Calculate the difference between the total number of deaths as provided by the NCHS Sex Data and the total number of deaths contained in intervals with non-suppressed death counts; this is the number of deaths contained in the union of intervals with suppressed death counts.

2. Let $\mathcal{B}$ denote the total number of $\mathrm{MC}$ iterations, and let $b=1, \ldots, \mathcal{B}$ index the $\mathrm{MC}$ iterations. For the task of obtaining a maximum MC point estimate of the estimand of interest that primarily concerns racial/ethnic group $r$ at each $\mathrm{MC}$ iteration $b$, combine the Unknown racial/ethnic group with racial/ethnic group $r$ into a combined $r$ Unknown racial/ethnic group whose constituents we all assume to be in racial/ethnic group $r$, combine the remaining racial/ethnic groups that are not included in the definition of the estimand into a single "other" racial/ethnic group, and enumerate all possible mortality datasets, omitting those that would yield a maximum MC point estimate with probability 0 . For the task of obtaining a minimum MC point estimate of the estimand of interest, combine all of the racial/ethnic groups not included in the definition of the estimand (including the Unknown racial/ethnic group) and enumerate all possible mortality datasets, omitting those that would yield a minimum MC point estimate with probability 0 . Let $J_{s}^{(\max )}$ denote the number of mortality datasets considered for the maximization task in state $s$, and let $J_{s}^{(\min )}$ denote the number of mortality datasets considered for the minimization task in state $s$.

3. Specify a YPLL upper reference age $\mathcal{A}$ less than or equal to 85 years. We view age group $<1$ as equivalent to the singular age 0 , and the remaining numeric NCHS age group endpoints represent integer age at last birthday so that there is a 1-year gap between the endpoints of two chronologically consecutive age groups (e.g., 35- 
44 and 45-54). We treat age as a continuous variable, and as a consequence, we mathematically interpret the $<1$ age group (age 0 ) as the right half-open interval $[0,1$ ), the $85+$ age group as the half-bounded interval $[85, \infty)$, and the remaining NCHS age groups as right half-open intervals with lower limit equal to the lower endpoint of the corresponding NCHS age group and upper limit equal to the upper endpoint of the corresponding NCHS age group plus one (e.g., age group 5-14 is viewed as $[5,15))$. At each MC iteration $b$ and for each mortality dataset $j=1, \ldots, J_{s}^{(\max )}+J_{s}^{(\min )}$ considered for the maximization and minimization tasks, independently simulate an age at death $\tilde{a}_{i j s}^{(b)}$ for each individual fatality $i$ corresponding to age interval $\left(L_{i j s}, U_{i j s}\right)$, where $\mathcal{A}>L_{i j s}$, from the corresponding continuous uniform distribution:

$$
\tilde{a}_{i j s}^{(b)} \stackrel{\text { ind }}{\sim} \mathcal{U}\left(L_{i j s}, U_{i j s}\right)
$$

Observe that $\mathcal{A}$ is intentionally and necessarily chosen to be less than or equal to 85 years to obviate the simulation of ages at death corresponding to the 85+ age group, thereby avoiding potential analytic difficulties because each fatality corresponding to the 85+ age group contributes nothing to YPLL.

4. At each MC iteration $b$ and for each mortality dataset $j$, calculate a point estimate of the estimand of interest. In particular, for the estimation of the percentage of total YPLL for racial/ethnic group $r$, first calculate total YPLL for racial/ethnic group $r$ (which includes the Unknown racial/ethnic group for the maximization task) and the remaining racial/ethnic groups from the simulated ages at death, which are $\widehat{\mathrm{YPLL}}_{r j s}^{(b)}=\sum_{i \in r} \max \left\{\mathcal{A}-\tilde{a}_{i j s}^{(b)}, 0\right\}$ and $\widehat{\mathrm{YPLL}}_{\bar{r} j s}^{(b)}=\sum_{i \notin r} \max \left\{\mathcal{A}-\tilde{a}_{i j s}^{(b)}, 0\right\}$, respectively. Then, the percentage of total YPLL for racial/ethnic group $r$, which we denote $\widehat{\pi}_{r j s}^{(b)}$, is given by:

$$
\widehat{\pi}_{r j s}^{(b)}=\frac{\widehat{\mathrm{YPLL}}_{r j s}^{(b)}}{\widehat{\mathrm{YPLL}}_{r j s}^{(b)}+\widehat{\mathrm{YPLL}}_{\bar{r} j s}^{(b)}} \times 100 \%
$$

For estimation of the age-adjusted $r$-to-NH White YPLL RR, first estimate the ageadjusted YPLL rates for racial/ethnic group $r$ and NH Whites, using the 2019 CDC WONDER age distribution estimate of the overall U.S. population as the standard population. The age-adjusted YPLL rate for racial/ethnic group $r$ (which includes the Unknown racial/ethnic group for the maximization task) is calculated using direct age adjustment [54] from the simulated ages at death, which we denote $\widehat{R}_{Y P L L, r j s}^{(b)}$. Since the simulated ages at death are continuous and the CDC WONDER age distribution estimates are defined over integer ages from 0 to 84, we aggregate the corresponding simulated YPLL values with respect the 1-year intervals implied by these integer ages (i.e., age $a \in\{0,1, \ldots, 84\}$ implies age interval $[a, a+1)$ ) to calculate the age-specific YPLL rates, which are subsequently applied to the standard population to obtain the age-adjusted YPLL rate for racial/ethnic group $r$ :

$$
\widehat{\mathrm{R}}_{\mathrm{YPLL}, r j s}^{(b)}=\frac{\sum_{a=0}^{84}\left(n_{a} \times \frac{\widehat{\mathrm{YPLL}}_{r j s a}^{(b)}}{n_{r s a}}\right)}{\sum_{a=0}^{84} n_{a}+n_{85+}}
$$


where $\widehat{\mathrm{YPLL}}_{r j s a}^{(b)}=\sum_{\substack{i \in r \\ \tilde{a}_{i j s}^{(b)} \in[a, a+1)}} \max \left\{\mathcal{A}-\tilde{a}_{i j s}^{(b)}, 0\right\}$ denotes aggregate YPLL correspond-

ing to age $a \in\{0,1, \ldots, 84\} ; n_{r s a}$ denotes the 2019 CDC WONDER U.S. population estimate for race/ethnicity $r$, state $s$, and age $a \in\{0,1, \ldots, 84\}$; and $n_{a}$ denotes the 2019 CDC WONDER U.S. population estimate for age $a \in\{0,1, \ldots, 84,85+\}$. Then, the age-adjusted $r$-to-NH White YPLL RR is defined to be the quotient of $\widehat{R}_{\mathrm{YPLL}, r j s}^{(b)}$ and $\widehat{\mathrm{R}}_{\mathrm{YPLL}, \mathrm{NH}}^{(b)}$ White, $j$ :

$$
\widehat{\operatorname{RR}}_{\mathrm{YPLL}, r, \mathrm{NH} \text { White }, j s}^{(b)}=\frac{\widehat{\mathrm{R}}_{\mathrm{YPLL}, r j s}^{(b)}}{\widehat{\mathrm{R}}_{\mathrm{YPLL}, \mathrm{NH} \text { White }, j s}^{(b)}} .
$$

5. At each MC iteration $b$, store the maximum of the $J_{s}^{(\max )} \mathrm{MC}$ point estimates of the estimand of interest calculated from the set of $J_{s}^{(\max )}$ mortality datasets considered for the maximization task, and store the minimum of the $J_{s}^{(\mathrm{min})} \mathrm{MC}$ point estimates calculated from the set of $J_{s}^{(\mathrm{min})}$ mortality datasets considered for the minimization task.

6. A conservative $(1-\alpha) \times 100 \%$ interval estimate of the estimand of interest is given by the $\frac{\alpha}{2}$ quantile of the $\mathcal{B}$ minimum MC point estimates and the $1-\frac{\alpha}{2}$ quantile of the $\mathcal{B}$ maximum $\mathrm{MC}$ point estimates.

Defining a valid overall point estimate for the estimand of interest is not straightforward due to our estimation strategy; to be explicit, the midpoint of the conservative $(1-\alpha) \times 100 \%$ interval estimate should not be interpreted as the point estimate. As such, in the results we present, we communicate our estimates solely as intervals, basing conclusions and interpretations on the ensemble of interval estimates we produce.

\subsection{Monte Carlo Simulation Procedure for Estimation of Age-Adjusted Mortality Rates and Rate Ratios}

Because we can enumerate the death count combinations for the intervals with suppressed death counts in each state, we can determine the entire plausible range of total deaths and percentage of total deaths by race/ethnicity in each state, which complement our estimates of total YPLL and percentage of total YPLL by race/ethnicity. At the time of writing, every U.S. state except North Dakota publicly reports COVID-19 death counts by race/ethnicity [55], which are more up to date than the (lagged) NCHS Race/Ethnicity Data. However, because different states can use different race/ethnicity categories, rendering direct comparisons between states difficult, we did not use the COVID-19 death counts by race/ethnicity that states themselves publicly report in our analysis. For comparison to our estimates of the age-adjusted YPLL rates and RR's, we also consider estimation of the corresponding age-adjusted mortality rates and RR's. We want these quantities to be age-standardized to the 2019 CDC WONDER age distribution estimate of the overall U.S. population-without combining CDC WONDER age intervals to align them with the NCHS age intervals - so that estimated YPLL and mortality rates in our analysis are age-standardized to as identical as possible standard populations in terms of age interval granularity. To this end, we perform an analogous $\mathrm{MC}$ simulation procedure to obtain conservative $(1-\alpha) \times 100 \%$ interval estimates of the age-adjusted mortality rates and RR's in the U.S. and in each examined state. The procedure largely mirrors the MC simulation procedure for the YPLL-based estimands described previously in Section 2.6. However, ages at death are simulated for all individuals in non-85+ age groups. In the direct age adjustment procedure, we sum the number of simulated ages at death falling within the 1-year intervals implied by integer ages 0 to 84 to calculate the age-specific mortality rates for ages 0 to 84 as well as calculate an age $85+$ mortality rate, which are subsequently 
applied to the standard population to obtain the age-adjusted mortality rate, which we denote $\widehat{\mathrm{R}}_{\text {mort }, r j s}^{(b)}$ for racial/ethnic group $r$ :

$$
\widehat{\mathrm{R}}_{\mathrm{mort}, r j \mathrm{~s} s}^{(b)}=\frac{\sum_{a=0}^{84}\left(n_{a} \times \frac{\hat{d}_{r j a s}^{(b)}}{n_{r j s a}}\right)+\left(n_{85+} \times \frac{\hat{d}_{r j, 85+}}{n_{r j, 85+}}\right)}{\sum_{a=0}^{84} n_{a}+n_{85+}}
$$

where $\hat{d}_{r j s a}^{(b)}=\sum_{\substack{i \in r \\ \tilde{a}_{i j s} \in[a, a+1)}} 1$ is the number of simulated ages at death equal to age $a \in\{0,1, \ldots, 84\}$, and $\hat{d}_{r j s, 85+}$ denotes the number of deaths in the $85+$ age group. Then, the age-adjusted $r$-to-NH White mortality RR is defined to be the quotient of $\widehat{\mathrm{R}}_{\text {mort,rjs }}^{(b)}$ and $\widehat{\mathrm{R}}_{\text {mort,NH White, } \mathrm{j} \text { : }}^{(b)}$

$$
\widehat{\mathrm{RR}}_{\text {mort }, r, \mathrm{NH} \text { White }, j s}^{(b)}=\frac{\widehat{\mathrm{R}}_{\text {mort }, r j s}^{(b)}}{\widehat{\mathrm{R}}_{\text {mort }, \mathrm{NH} \text { White }, j s}^{(b)}} .
$$

\subsection{Computation}

We perform the modified $\mathrm{Xu}$ et al. MC simulation procedure described in Section 2.6 for $\mathcal{B}=1000$ iterations using a constant YPLL upper reference age of $\mathcal{A}=75$ years for all racial/ethnic groups considered, an approach used by the Centers for Disease Control and Prevention (CDC) [56-63] and in other applied research domains [64,65], obtaining conservative 95\% interval estimates of total YPLL, percentage of total YPLL, and ageadjusted YPLL rates by race/ethnicity as well as age-adjusted YPLL RR's for NH Blacks, Hispanics, NH Asians, and NH AIAN's relative to NH Whites in each of the 46 examined states. An alternative approach that has been employed in applied studies contrasting YPLL by race/ethnicity is to use race/ethnicity-specific YPLL upper reference ages corresponding to the respective life expectancies $[36,37,66-69]$, thereby reflecting known heterogeneity in underlying life expectancies between racial/ethnic groups. Using race/ethnicity-specific values of $\mathcal{A}$, however, would reduce the magnitudes of any disparities and could even reverse their direction compared to results obtained using a constant value of $\mathcal{A}$ for all racial/ethnic groups as a result of implicitly "setting a lower bar" for the total potential years of life of racial/ethnic minorities, which could potentially lead to misinterpretation of study findings. Out of a desire informed by a health equity perspective not to necessarily normalize underlying racial/ethnic disparities in life expectancy, a sentiment shared in other applications [70], we decided to use a constant YPLL upper reference age $(\mathcal{A}=75)$ for all racial/ethnic groups considered to provide a comparison of YPLL by race/ethnicity that is not dependent on underlying racial/ethnic differences in life expectancies. We also obtain conservative $95 \%$ interval estimates of age-adjusted mortality rates by race/ethnicity as well as age-adjusted mortality RR's for NH Blacks, Hispanics, NH Asians, and NH AIAN's relative to NH Whites using an analogous MC simulation procedure described in Section 2.7, also for $\mathcal{B}=1000$ iterations. All MC simulations were performed using the $\mathrm{R}$ version 3.6.0 programming language [71]. The code used in our analysis is available upon reasonable request from the corresponding author. 


\section{Results}

Tables S1-S5 in the Supplementary Materials contain the entirety of the results. Table S1 presents conservative 95\% interval estimates of total COVID-19-attributable YPLL for the $5 \mathrm{racial} / \mathrm{ethnic}$ groups considered in the U.S. and in each of the 46 examined states, and Table S2 presents intervals denoting the entire plausible range of total COVID-19 deaths by race/ethnicity in the U.S. and in each examined state. Table S3 presents conservative 95\% interval estimates of the percentage of total COVID-19-attributable YPLL and intervals denoting the entire plausible range of the percentage of total COVID-19 deaths for the 5 racial/ethnic groups considered in the U.S. and in each of the examined states. When the interval of the percentage of total deaths for racial/ethnic group $r \in\{\mathrm{NH}$ White, NH Black, Hispanic, NH Asian, NH AIAN $\}$ is completely above its percent population share, racial/ethnic group $r$ is overrepresented among COVID-19 deaths. Conversely, when the interval of the percentage of total deaths for racial/ethnic group $r$ is completely below its percent population share, racial/ethnic group $r$ is underrepresented among COVID-19 deaths. The interpretation of the interval estimates of the percentage of total YPLL relative to the percent population share is slightly more nuanced, however. When the interval estimate of the percentage of total YPLL for racial/ethnic group $r$ is completely above its percent population share, racial/ethnic group $r$ is either overrepresented among COVID-19 deaths or decedent ages among racial/ethnic group $r$ are systematically younger relative to decedents of the other racial/ethnic groups-to a degree that is statistically discernable-or both. Conversely, when the interval estimate of the percentage of total YPLL for racial/ethnic group $r$ is completely below its percent population share, racial/ethnic group $r$ is either underrepresented among COVID-19 deaths or decedent ages among racial/ethnic group $r$ are systematically older relative to decedents of the other racial/ethnic groups-to a degree that is statistically discernable — or both.

The magnitudes of the disparities in the COVID-19 mortality burden can be amplified when mortality is measured in terms of YPLL compared to (age-irrespective) death counts. For example, if racial/ethnic group $r$ is overrepresented among COVID-19 deaths, the interval estimate of the percentage of total YPLL can additionally be completely above the percentage of total deaths as a result of decedent ages being systematically older relative to other racial/ethnic groups to a degree that is statistically discernable. Similarly, if racial/ethnic group $r$ is underrepresented among COVID-19 deaths, the interval estimate of the percentage of total YPLL can additionally be completely below the percentage of total deaths as a result of decedent ages being systematically older relative to other racial/ethnic groups to a degree that is statistically discernable.

Moreover, the direction of the disparity in the COVID-19 mortality burden can in fact reverse when mortality is measured in terms of YPLL compared to death counts. For example, if racial/ethnic group $r$ is underrepresented among COVID-19 deaths, the interval estimate of the percentage of total YPLL can, in contrast, be completely above the percent population share as a result of decedent ages being systematically younger relative to other racial/ethnic groups to a degree that is both statistically discernable and outweighs the disproportionately low number of deaths. Similarly, if racial/ethnic group $r$ is overrepresented among COVID-19 deaths, the interval estimate of the percentage of total YPLL can, in contrast, be completely below the percent population share as a result of decedent ages being systematically older relative to other racial/ethnic groups to a degree that is both statistically discernable and outweighs the disproportionately high number of deaths.

Table S4 presents conservative 95\% interval estimates of the age-adjusted YPLL and mortality rates per 10,000 population for the 5 racial/ethnic groups considered in the U.S. and in the 46 examined states. Table S5 presents conservative $95 \%$ interval estimates of the age-adjusted YPLL and mortality RR's for NH Blacks, Hispanics, $\mathrm{NH}$ Asians, and NH AIAN's relative to NH Whites in the U.S. and in the examined states. When the interval estimate of the age-adjusted $r$-to-NH White mortality RR $(r \in\{\mathrm{NH}$ Black, Hispanic, NH Asian, NH AIAN $\})$ is completely above 1.0, it means that 
after accounting for differences in the population age distributions between racial/ethnic group $r$ and NH Whites, racial/ethnic group $r$ experiences COVID-19-attributable death at a rate that that is statistically discernably above that of NH Whites. Similarly, when the interval estimate of the age-adjusted $r$-to-NH White YPLL RR is completely above 1.0 , it means that after accounting for differences in the population age distributions between racial/ethnic group $r$ and NH Whites, racial/ethnic group $r$ experiences COVID19-attributable YPLL at a rate that is statistically discernably above that of NH Whites. When the interval estimate of the age-adjusted $r$-to-NH White YPLL RR is completely above the interval estimate of the age-adjusted $r$-to-NH White mortality RR, the $r$-NH White disparity in the COVID-19 mortality burden is statistically discernably greater in magnitude when measuring mortality in terms of YPLL compared to death counts as a result of individuals of racial/ethnic group $r$ dying at systematically and statistically discernably younger ages relative to NH Whites after accounting for differences in their population age distributions.

\subsection{Results for Non-Hispanic Whites}

Figure 1 displays a graphical comparison between the conservative 95\% interval estimates of the percentage of total YPLL, intervals denoting the entire plausible range of the percentage of total deaths, and the percent population share for NH Whites in the U.S. and in each examined state. Nationally, NH Whites are underrepresented among COVID-19 deaths, comprising $57.6-58.1 \%$ of total deaths despite representing $61.2 \%$ of the U.S. population. At the state level, NH Whites are overrepresented among COVID19 deaths in 24 of the examined states but are underrepresented in 21 of the examined states. However, NH White COVID-19 decedent ages in the U.S. overall are systematically older relative the other racial/ethnic groups to such a degree that the U.S. national NH White conservative 95\% interval estimate of the percentage of total YPLL ([40.1-40.8\%]) is completely below the U.S. national NH White percentage of total deaths. At the state level, the interval estimates of the percentage of total YPLL are completely below the percentages of total deaths in 44 of the 46 examined states, reflecting a consistent pattern across states of NH Whites dying from COVID-19 at older ages than individuals of other racial/ethnic groups. For example, in Louisiana, NH Whites represent $59.1 \%$ of the population and only $52.6-53.4 \%$ of total deaths, but the interval estimate of the percentage of total YPLL is an even lower [29.7-34.7\%]. Furthermore, interval estimates of the percentage of total YPLL are completely below the NH White percent population share in 43 of the 46 examined states, greater than the corresponding number for the percentages of total deaths. The direction of the disparity in fact reverses when using YPLL rather than death counts to measure mortality in 21 of the 24 states where $\mathrm{NH}$ Whites are overrepresented among COVID-19 deaths. For example, in Massachusetts, NH Whites represent $72.1 \%$ of the population and constitute a disproportionately high $80.1-81.3 \%$ of total deaths, but the interval estimate of the percentage of total YPLL is only [54.0-62.7\%].

\subsection{Results for Non-Hispanic Blacks}

Figure 2 displays a graphical comparison between the conservative 95\% interval estimates of the percentage of total YPLL, intervals denoting the entire plausible range of the percentage of total deaths, and the percent population share for NH Blacks in the U.S. and in each examined state. Nationally, NH Blacks are overrepresented among COVID-19 deaths, comprising $16.9-17.4 \%$ of total deaths despite representing only $13.2 \%$ of the U.S. population. Indeed, at the state level, NH Blacks are overrepresented among COVID19 deaths in 26 of the examined states and underrepresented in only 8 of the examined states. In fact, the NH Blacks comprise the greatest percentage of total COVID-19 deaths in D.C, although this is partially due to the fact that NH Blacks are the predominant racial/ethnic group in D.C., comprising over $45 \%$ of the population. Nevertheless, the disparity between the NH Black percentage of total deaths and the NH Black percent population share is by far the highest in D.C. among the examined states. Furthermore, 
NH Black COVID-19 decedent ages in the U.S. overall are systematically younger relative to other racial/ethnic groups to such a degree that the U.S. national NH Black conservative 95\% interval estimate of the percentage of total YPLL ([23.0-23.6\%]) is completely above the U.S. national NH Black percentage of total deaths. Indeed, at the state level, interval estimates of the percentage of total YPLL are above the percentages of total deaths in 27 of the examined states and completely below in none of the examined states. For example, $\mathrm{NH}$ Blacks represent $14.6 \%$ of the population in Michigan but an astonishing 27.7-29.0\% of total deaths, yet the interval estimate of the percentage of total YPLL is a staggering [42.4-47.4\%]. Interestingly, the reverse phenomenon is observed in D.C., where the interval estimate of the percentage of total YPLL ([52.3-68.9\%]) is completely below the percentage of total deaths ([69.6-71.4\%]). Interval estimates of the percentage of total YPLL are completely above the NH Black percent population share in 33 of the 46 examined states, greater than the corresponding number for the percentages of total deaths, and completely below in none of the examined states. Furthermore, interval estimates of the percentage of total YPLL are completely above all of the corresponding intervals for the other racial/ethnic groups in 6 of the examined states (D.C., Georgia, Louisiana, Maryland, Mississippi, and South Carolina). Moreover, the direction of the disparity in fact reverses when using YPLL rather than death counts to measure mortality in 3 of the 8 states (Arkansas, Iowa, and Minnesota) where NH Blacks are underrepresented among COVID-19 deaths. For example, in Minnesota, NH Blacks represent $7.5 \%$ of the population and only $5.3-6.2 \%$ of total deaths, but the interval estimate of the percentage of total YPLL is [12.9-21.7\%].

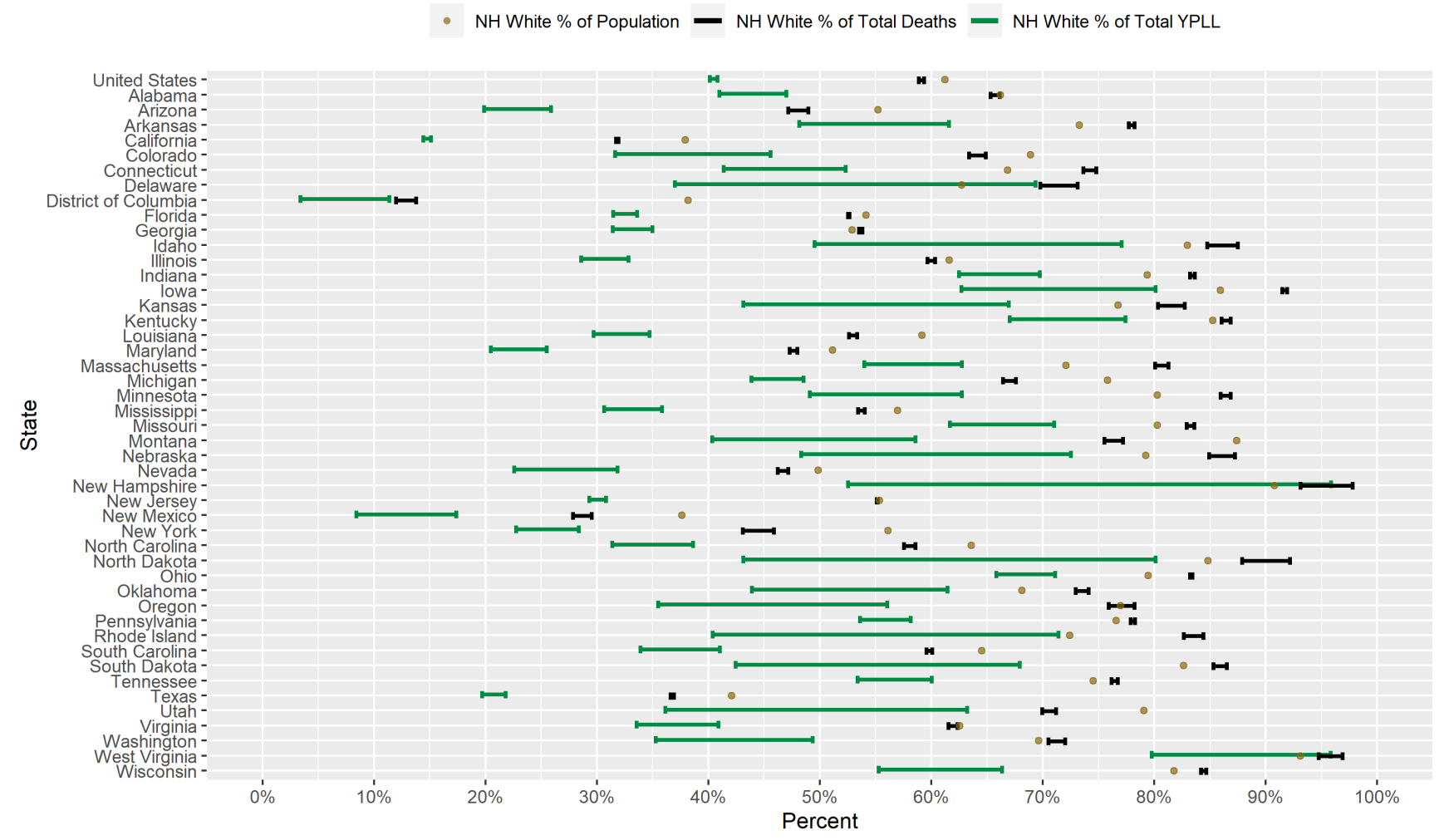

Figure 1. Conservative 95\% interval estimates of the percentage of total COVID-19-attributable YPLL before age 75, intervals denoting the entire plausible range for the percentage of total COVID-19 deaths, and the percent population shares for NH Whites in the U.S. and in each examined state with respect to cumulative COVID-19 deaths according to data from the National Center for Health Statistics as of 30 December 2020. 


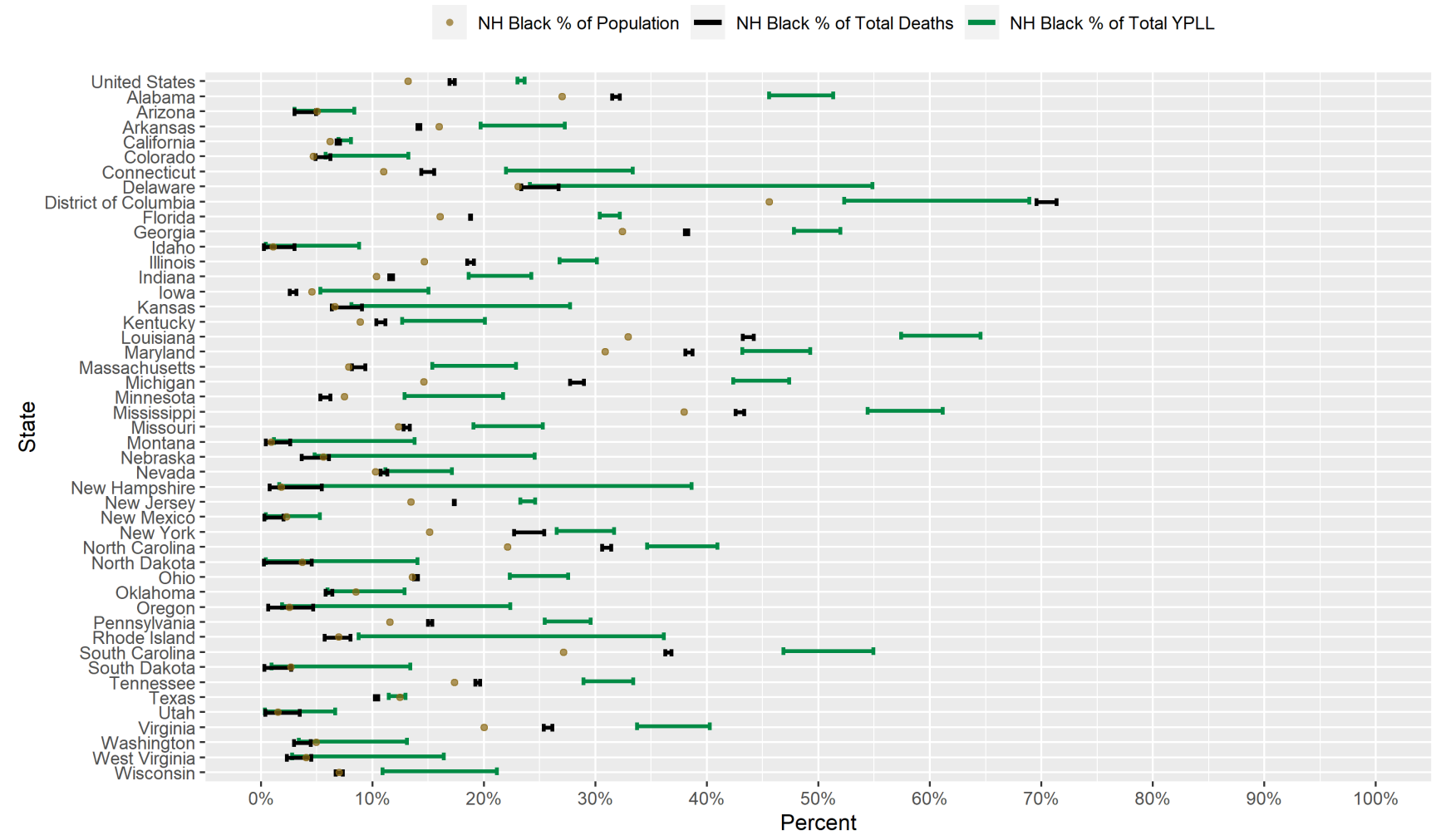

Figure 2. Conservative 95\% interval estimates of the percentage of total COVID-19-attributable YPLL before age 75, intervals denoting the entire plausible range for the percentage of total COVID-19 deaths, and the percent population shares for $\mathrm{NH}$ Blacks in the U.S. and in each examined state with respect to cumulative COVID-19 deaths according to data from the National Center for Health Statistics as of 30 December 2020.

Figure 3 presents a graphical comparison of the conservative 95\% interval estimates of the age-adjusted NH Black-to-NH White YPLL and mortality RR's in the U.S. and in each examined state. The U.S. national conservative $95 \%$ interval estimate of the ageadjusted NH Black-to-NH White mortality RR is [1.91-1.99], with the state-level mortality RR interval estimates completely above 1.0, 2.0, and 3.0 in 39, 17, and 2 of the examined states, respectively. However, NH Black decedent ages in the U.S. overall are systematically younger relative to NH Whites, after accounting for differences in the national population age distributions between NH Blacks and NH Whites, to such a degree that the U.S. national conservative 95\% interval estimate of the age-adjusted NH Black-to-NH White YPLL RR is [2.84-2.97], completely above the U.S. national mortality RR interval estimate. Indeed, in 30 of the 46 examined states, the YPLL RR interval estimates are completely above the mortality RR interval estimates, reflecting a broad pattern across states of NH Blacks dying from COVID-19 at earlier ages than NH Whites after accounting for differences in their respective state population age distributions. Moreover, state-level YPLL RR interval estimates are completely above 1.0, 2.0 and 3.0 in 39, 33, and 17 of the examined states, respectively, greater than or equal to the corresponding numbers for the state-level mortality RR interval estimates. Among the examined states, Michigan stands out markedly with the highest age-adjusted NH Black-NH White disparity in COVID-19-attributable YPLL with a YPLL RR interval estimate of [5.77-6.67]. 


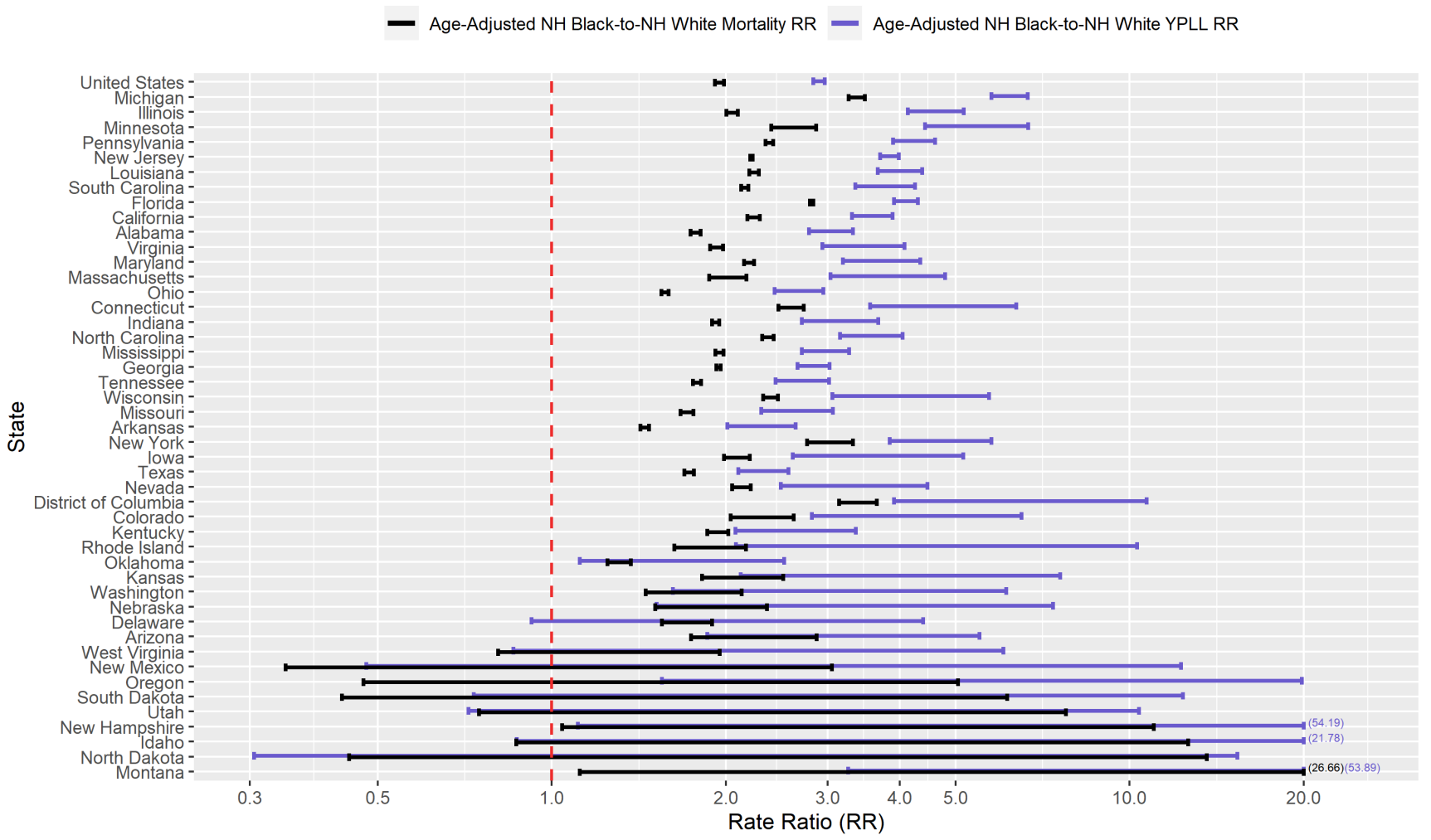

Figure 3. Conservative 95\% interval estimates of the age-adjusted NH Black-to-NH White YPLL and mortality RR's in the U.S. and in each examined state with respect to cumulative COVID-19 deaths according to data from the National Center for Health Statistics as of 30 December 2020. States are ordered from top to bottom in descending order of the signed difference between the lower limit of the YPLL RR interval and the upper limit of the mortality RR interval. Values are displayed on the base 10 logarithmic scale. Interval endpoints above 20.0 and below 0.30 are truncated, with the actual values numerically annotated.

\subsection{Results for Hispanics}

Figure 4 displays a graphical comparison between the conservative $95 \%$ interval estimates of the percentage of total YPLL, intervals denoting the entire plausible range of the percentage of total deaths, and the percent population share for Hispanics in the U.S. and in each examined state. Nationally, COVID-19 deaths among Hispanics are approximately proportional to their percent population share: Hispanics comprise $18.5 \%$ of the U.S. population and 18.6-19.1\% of COVID-19 deaths. At the state level, Hispanics are overrepresented among COVID-19 deaths in 11 of the examined states and underrepresented in 29 of the examined states. Hispanics comprise a greater percentage of COVID-19 deaths than NH Whites in 4 of the examined states (California, D.C., New Mexico, and Texas) and comprise the greatest percentage of COVID-19 deaths among the racial/ethnic groups considered in 2 of those states (California and Texas). Although COVID-19 deaths among Hispanics nationally are about in line with their percent population share, Hispanic COVID-19 decedent ages in the U.S. overall are systematically younger relative to non-Hispanics to such a degree that the U.S. national Hispanic conservative 95\% interval estimate of the percentage of total YPLL ([30.3-31.0\%]) is completely above the U.S. national Hispanic percentage of total deaths. This national trend of Hispanics dying from COVID-19 at systematically earlier ages relative to non-Hispanics is also widely observed at the state level, with the interval estimates of the percentage of total YPLL completely above the percentages of total deaths in 38 of the examined states and completely below in none of the examined states. For example, in Illinois, Hispanics represent $17.5 \%$ of the population and comprise a commensurate $17.3-18.0 \%$ of total deaths, but the interval estimate of the percentage of total YPLL is a much higher [35.5-39.5\%]. 


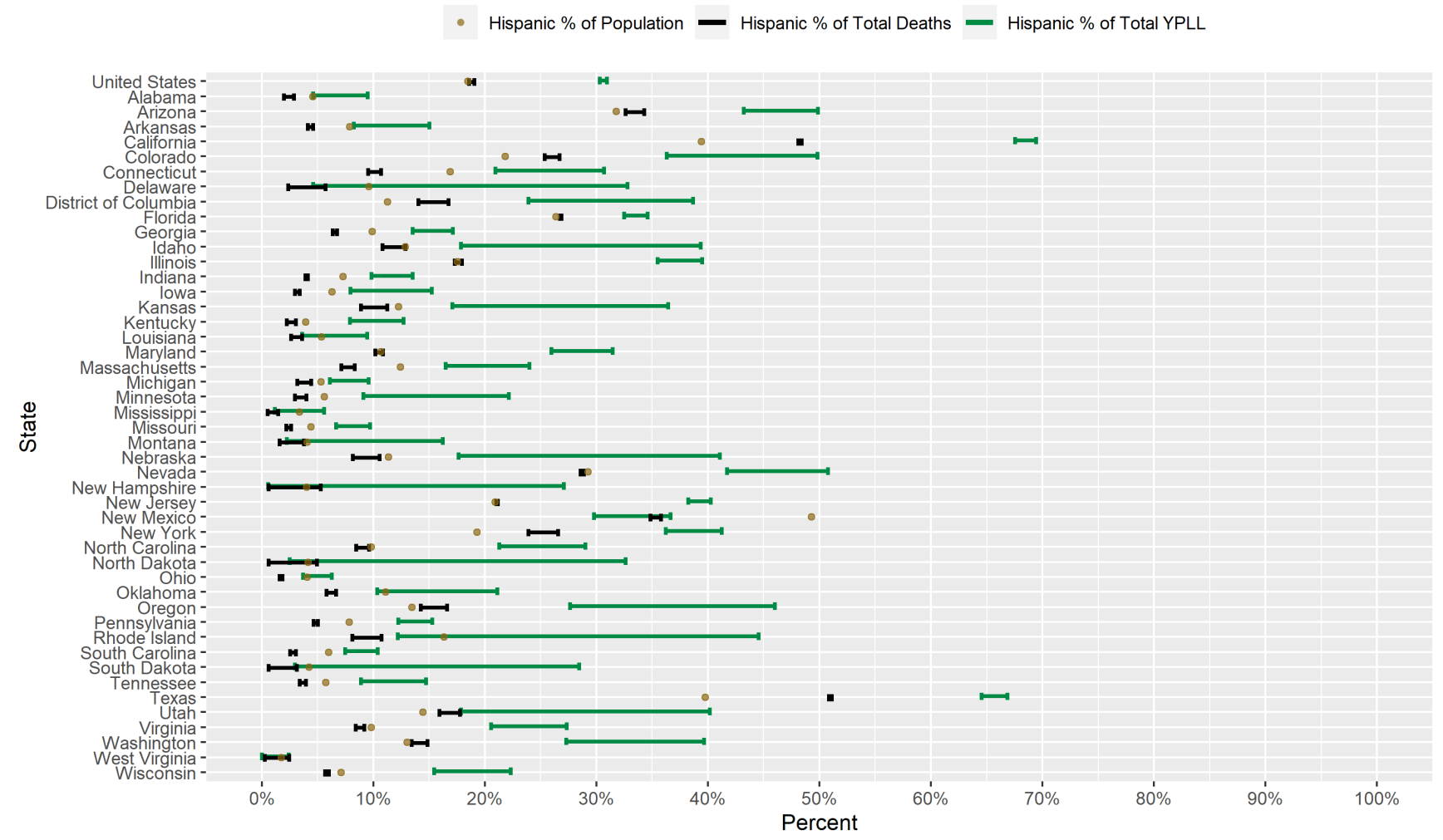

Figure 4. Conservative 95\% interval estimates of the percentage of total COVID-19-attributable YPLL before age 75, intervals denoting the entire plausible range for the percentage of total COVID-19 deaths, and the percent population shares for Hispanics in the U.S. and in each examined state with respect to cumulative COVID-19 deaths according to data from the National Center for Health Statistics as of 30 December 2020.

Interval estimates of the percentage of total YPLL are completely above the Hispanic percent population share in 34 out of the 46 examined states, greater than the corresponding number for the percentages of total deaths, and completely below in only 1 state (New Mexico). Furthermore, interval estimates of the percentage of total YPLL are completely above the corresponding intervals for $\mathrm{NH}$ Whites in 10 of the examined states and completely above all of the corresponding intervals for the other racial/ethnic groups in 7 of those states, greater than the corresponding numbers for the percentages of total deaths. The direction of the disparity in fact reverses when using YPLL rather than death counts to measure mortality in 20 of the 29 states where the Hispanics are underrepresented among COVID-19 deaths. For example, in Connecticut, Hispanics represent $16.9 \%$ of the population and only $9.5-10.7 \%$ of total deaths, but the interval estimate of the percentage of total YPLL is [20.9-30.7\%]. We also note that the results for Hispanics in New Mexico are anomalous compared to the rest of the examined states, with both the percentage of total deaths and the interval estimate of the percentage of total YPLL markedly below the Hispanic percent population share: Hispanics represent nearly $50 \%$ of the population in New Mexico but comprise only $34.9-35.8 \%$ of total deaths, and the interval estimate of the percentage of total YPLL is [29.8-36.7\%].

Figure 5 presents a graphical comparison of the conservative 95\% interval estimates of the age-adjusted Hispanic-to-NH White YPLL and mortality RR's in the U.S. and in each examined state. The U.S. national conservative $95 \%$ interval estimate of the ageadjusted Hispanic-to-NH White mortality RR is [1.95-2.03], with the state-level mortality RR interval estimates completely above 1.0, 2.0, and 3.0 in 40, 21, and 6 of the examined states, respectively. However, Hispanic COVID-19 decedent ages in the U.S. overall are systematically younger relative to $\mathrm{NH}$ Whites, after accounting for differences in the national population age distributions between Hispanics and NH Whites, to such a degree 
that the U.S. national conservative 95\% interval estimate of the age-adjusted Hispanic-toNH White YPLL RR is [2.90-3.02], completely above the U.S. national mortality RR interval estimate. Indeed, the YPLL RR interval estimates are completely above the mortality RR interval estimates in 37 of the 46 examined states and are completely below in none of the examined states, reflecting a robust pattern across states of Hispanics dying from COVID-19 at earlier ages than $\mathrm{NH}$ Whites after accounting for differences in their respective state population age distributions. Moreover, interval estimates of the age-adjusted Hispanic-toNH White YPLL RR are completely above 1.0, 2.0 and 3.0 in 42, 35, and 26 of the examined states, respectively, greater than the corresponding numbers for the state-level mortality RR estimates. The YPLL RR interval estimates are notably high and disconcerting in a sizable number of states: Illinois, Washington, and Wisconsin (completely above 5.0); California, North Carolina, and Oregon (completely above 6.0); Maryland (completely above 7.0); and D.C. (completely above 8.0).

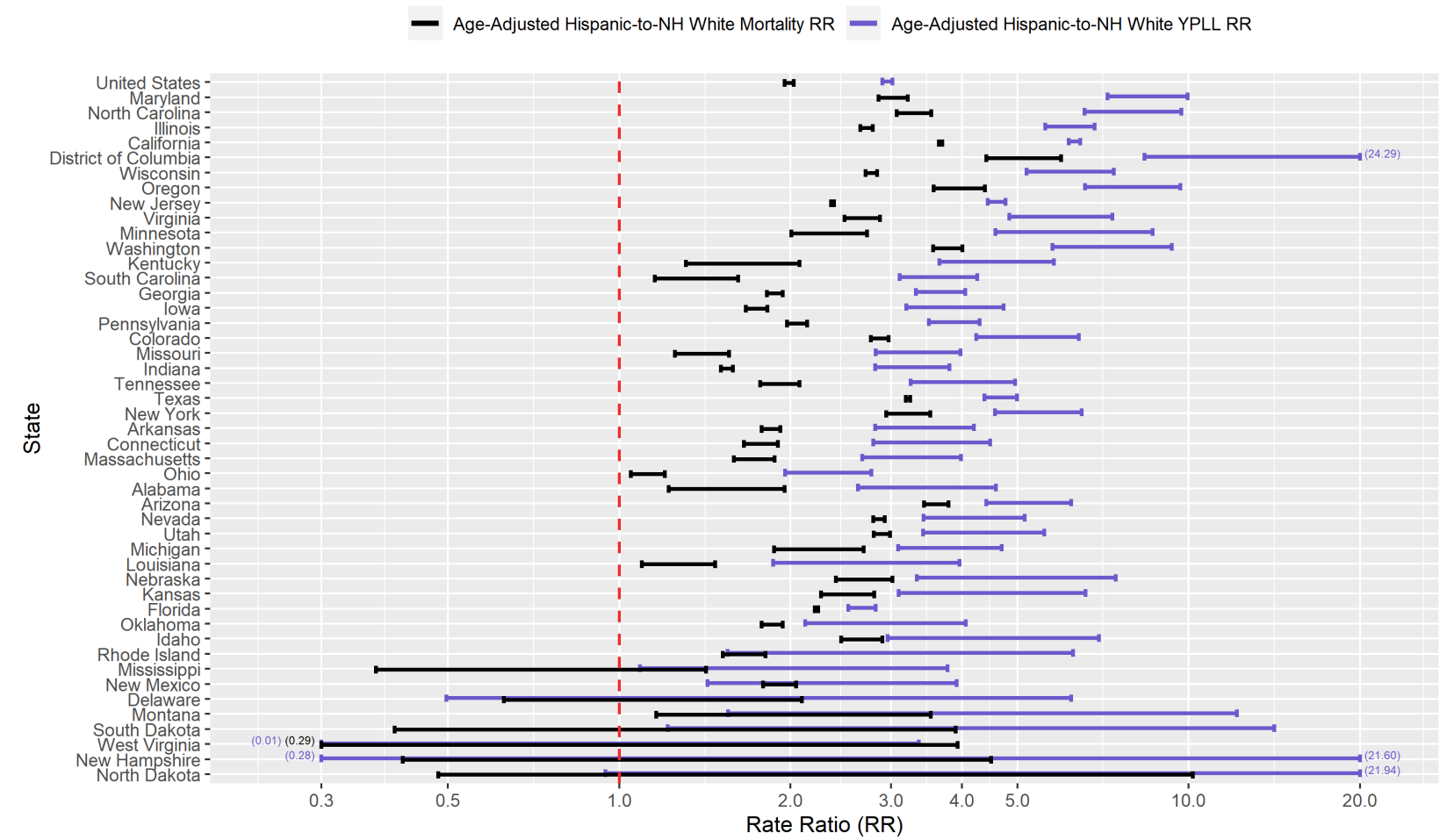

Figure 5. Conservative 95\% interval estimates of the age-adjusted Hispanic-to-NH White YPLL and mortality RR's in the U.S. and in each examined state with respect to cumulative COVID-19 deaths according to data from the National Center for Health Statistics as of 30 December 2020. States are ordered from top to bottom in descending order of the signed difference between the lower limit of the YPLL RR interval and the upper limit of the mortality RR interval. Values are displayed on the base 10 logarithmic scale. Interval endpoints above 20.0 and below 0.30 are truncated, with the actual values numerically annotated.

\subsection{Results for Non-Hispanic Asians}

Figure 6 displays a graphical comparison between the conservative 95\% interval estimates of the percentage of total YPLL, intervals denoting the entire plausible range of the percentage of total deaths, and the percent population share for NH Asians in the U.S. and in each examined state. Nationally, NH Asians are underrepresented among COVID-19 deaths, comprising only $3.6-4.0 \%$ of COVID-19 deaths despite representing $6.3 \%$ of the U.S. population. Accordingly, at the state level, NH Asians are underrepresented among COVID-19 deaths in 32 of the examined states and pverrerpesented in only 1 examined state (Nevada). The U.S. national NH Asian conservative 95\% interval estimate of the percentage of total YPLL ([3.8-4.4\%]) is statistically indistinguishable from the U.S. national 
percentage of total deaths, reflecting no detectable difference in COVID-19 decedent ages between NH Asians and the other racial/ethnic groups at the national level. However, in 3 of the examined states (Minnesota, Pennsylvania, and Wisconsin), the interval estimates of the percentage of total YPLL are completely above the percentage of total deaths, indicating that NH Asians die from COVID-19 at systematically and statistically discernably earlier ages than individuals of other racial/ethnic groups in these states. Conversely, in 2 of the examined states (California and Nevada), the interval estimates of the percentage of total YPLL are completely below the percentage of total deaths, indicating that $\mathrm{NH}$ Asians die from COVID-19 at systematically and statistically discernably older ages than individuals of other racial/ethnic groups in these states. Furthermore, interval estimates of the percentage of total YPLL contain the NH Asian percent population share in the vast majority of the examined states $(n=37)$, are completely above the $\mathrm{NH}$ Asian percent population share in 1 state (Minnesota), and are completely below the NH Asian percent population share in 8 states. In Minnesota, the direction of the disparity in fact reverses when using YPLL rather than death counts to measure mortality: NH Asians comprise $5.5 \%$ of the population and only $3.6-4.5 \%$ of total deaths, but the interval estimate of the percentage of total YPLL is [8.0-15.1\%]. The estimated ordered relationship between the $\mathrm{NH}$ Asian percent population share, percentage of total deaths, and percentage of total YPLL in California is unique among the 46 examined states: NH Asians represent $16.0 \%$ of the population and constitute only 11.6-11.9\% of total COVID-19 deaths, but the interval estimate of the percentage of total YPLL is an even lower [7.1-8.2\%], a result of NH Asians dying from COVID-19 at systematically and statistically discernably older ages relative to the other racial/ethnic groups.

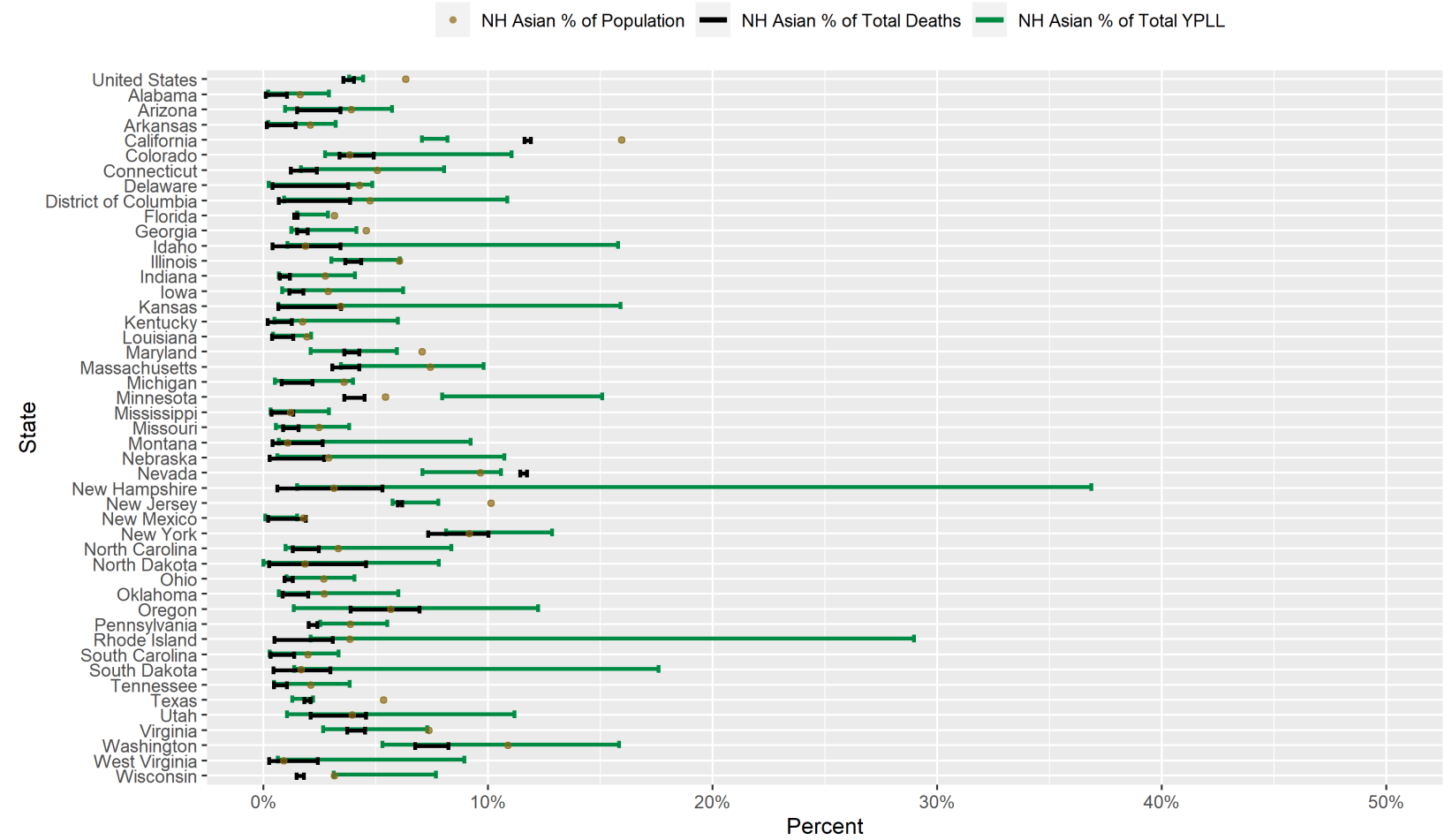

Figure 6. Conservative 95\% interval estimates of the percentage of total COVID-19-attributable YPLL before age 75, intervals denoting the entire plausible range for the percentage of total COVID-19 deaths, and the percent population shares for NH Asians in the U.S. and in each examined state with respect to cumulative COVID-19 deaths according to data from the National Center for Health Statistics as of 30 December 2020.

Figure 7 presents a graphical comparison of the conservative 95\% interval estimates of the age-adjusted NH Asian-to-NH White YPLL and mortality RR's in the U.S. and in each examined state. The U.S. national conservative $95 \%$ interval estimate of the age- 
adjusted NH Asian-to-NH White mortality RR is [0.83-0.95], meaning that after accounting for differences in the national population age distributions between $\mathrm{NH}$ Asians and $\mathrm{NH}$ Whites, NH Asians die from COVID-19 at a rate that is statistically discernably lower than that of NH Whites. At the state level, however, the state-level mortality RR interval estimates are completely above 1.0 in 14 of the examined states and completely below 1.0 in only 4 of the examined states. Moreover, NH Asian decedent ages in the U.S. overall are systematically younger relative to NH Whites, after accounting for differences in the national population age distributions between $\mathrm{NH}$ Asians and $\mathrm{NH}$ Whites, to such a degree that the U.S. national conservative 95\% interval estimate of the age-adjusted NH Asian-toNH White YPLL RR is [0.99-1.17], completely above the U.S. national mortality RR interval estimate and also nearly representing a statistically discernable reversal in the direction of the age-adjusted NH Asian-NH White disparity in the COVID-19 mortality burden. At the state level, however, the YPLL RR interval estimates are completely above the mortality RR interval estimates in only 4 of the 46 examined states (California, Minnesota, New Jersey, and Wisconsin) and completely below in none of the examined states, suggesting that the age-adjusted national trend of NH Asians dying from COVID-19 at earlier ages than NH Whites is driven in large part by a small subset of states. Furthermore, state-level YPLL RR interval estimates are completely above 1.0 in 11 of the examined states, slightly below the corresponding number for state-level mortality RR interval estimates, but completely above 2.0 in 2 of the examined states (Minnesota and Wisconsin). In particular, the YPLL RR interval estimate in Minnesota ([3.82-6.05]) stands out markedly among the examined states with the highest age-adjusted NH Asian-NH White disparity in COVID-19-attributable YPLL.

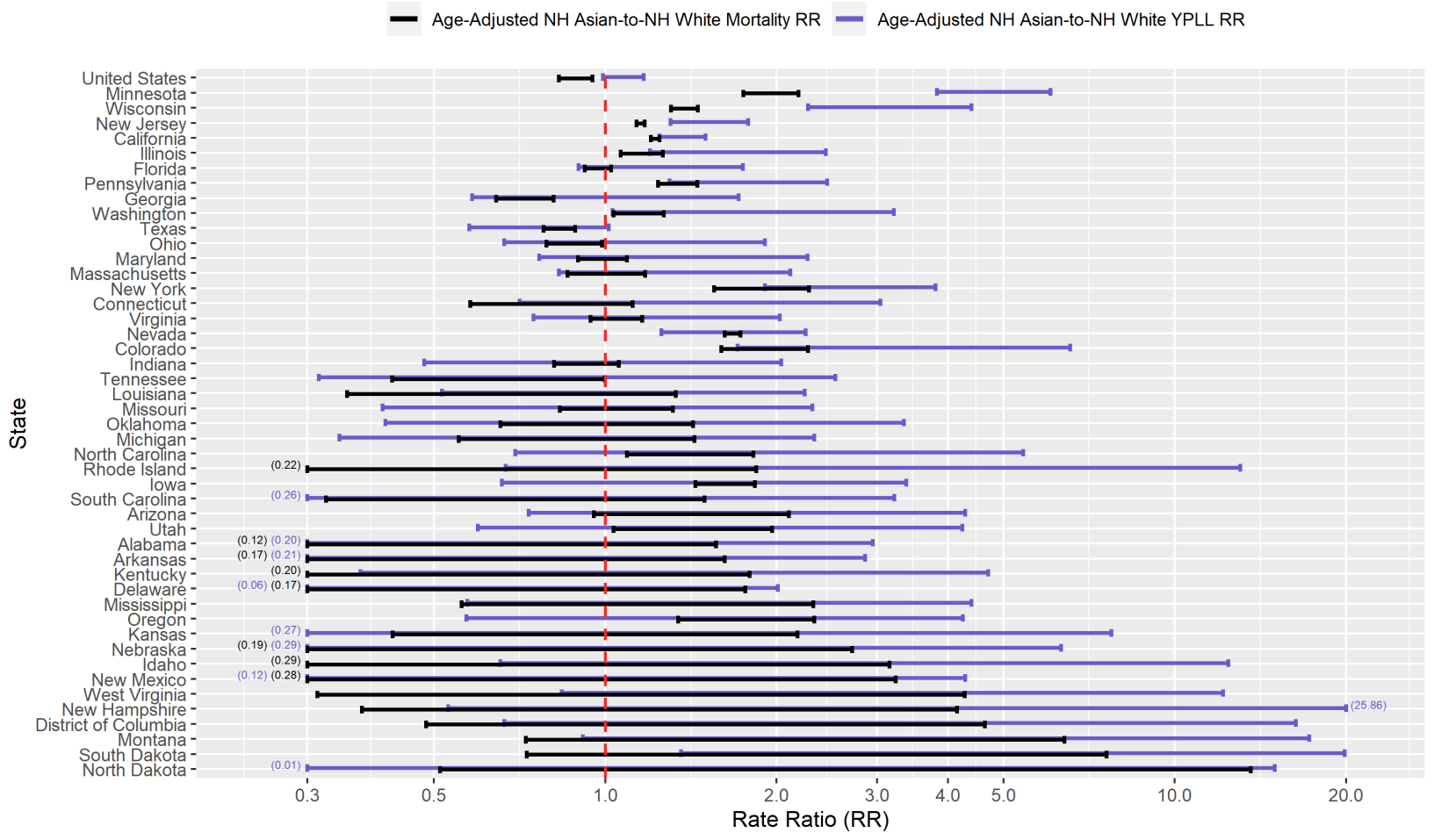

Figure 7. Conservative 95\% interval estimates of the age-adjusted NH Asian-to-NH White YPLL and mortality RR's in the U.S. and in each examined state with respect to cumulative COVID-19 deaths according to data from the National Center for Health Statistics as of 30 December 2020. States are ordered from top to bottom in descending order of the signed difference between the lower limit of the YPLL RR interval and the upper limit of the mortality RR interval. Values are displayed on the base 10 logarithmic scale. Interval endpoints above 20.0 and below 0.30 are truncated, with the actual values numerically annotated. 


\subsection{Results for Non-Hispanic American Indian or Alaska Natives}

Figure 8 displays a graphical comparison between the conservative $95 \%$ interval estimates of the percentage of total YPLL, intervals denoting the entire plausible range of the percentage of total deaths, and the percent population share for NH AIAN's in the U.S. and in each examined state. Nationally, NH AIAN's are overrepresented among COVID-19 deaths, comprising 1.1-1.6\% of COVID-19 deaths despite representing only $0.84 \%$ of the U.S. population. At the state level, NH AIAN's are overrepresented among COVID-19 deaths in 12 of the examined states and underrepresented in only 1 examined state (Florida). However, NH AIAN COVID-19 decedent ages in the U.S. overall are systematically younger relative to the other racial/ethnic groups to such a degree that the U.S. national NH AIAN conservative 95\% interval estimate of the percentage of total YPLL is [2.0-2.6\%], completely above the NH AIAN national percentage of total deaths. Moreover, at the state level, the interval estimates of the percentage of total YPLL are completely above the percentages of total deaths in 10 of the examined states and completely below in none of the examined states. For example, in South Dakota, NH AIAN's represent $8.8 \%$ of the population and $10.6-12.4 \%$ of total deaths, but the interval estimate of the percentage of total YPLL is an even greater [20.4-45.5\%]. The interval estimates of the percentage of total YPLL are completely above the NH AIAN percent population share in 15 of the examined states, greater than the corresponding number for the NH AIAN percentages of total deaths, and completely below in none of the examined states. The magnitudes of the disparities in the COVID-19 mortality burden for NH AIAN's are shockingly immense in 3 of the examined states (Arizona, Montana, and New Mexico), with both the percentages of total deaths and interval estimates of the percentage of total YPLL exorbitantly exceeding the NH AIAN percent population share. For example, NH AIAN's represent only $9.1 \%$ of the population of New Mexico but a stunning 34.8-35.7\% of total deaths, yet the interval estimate of the percentage of total YPLL is a staggering [48.5-57.0\%].

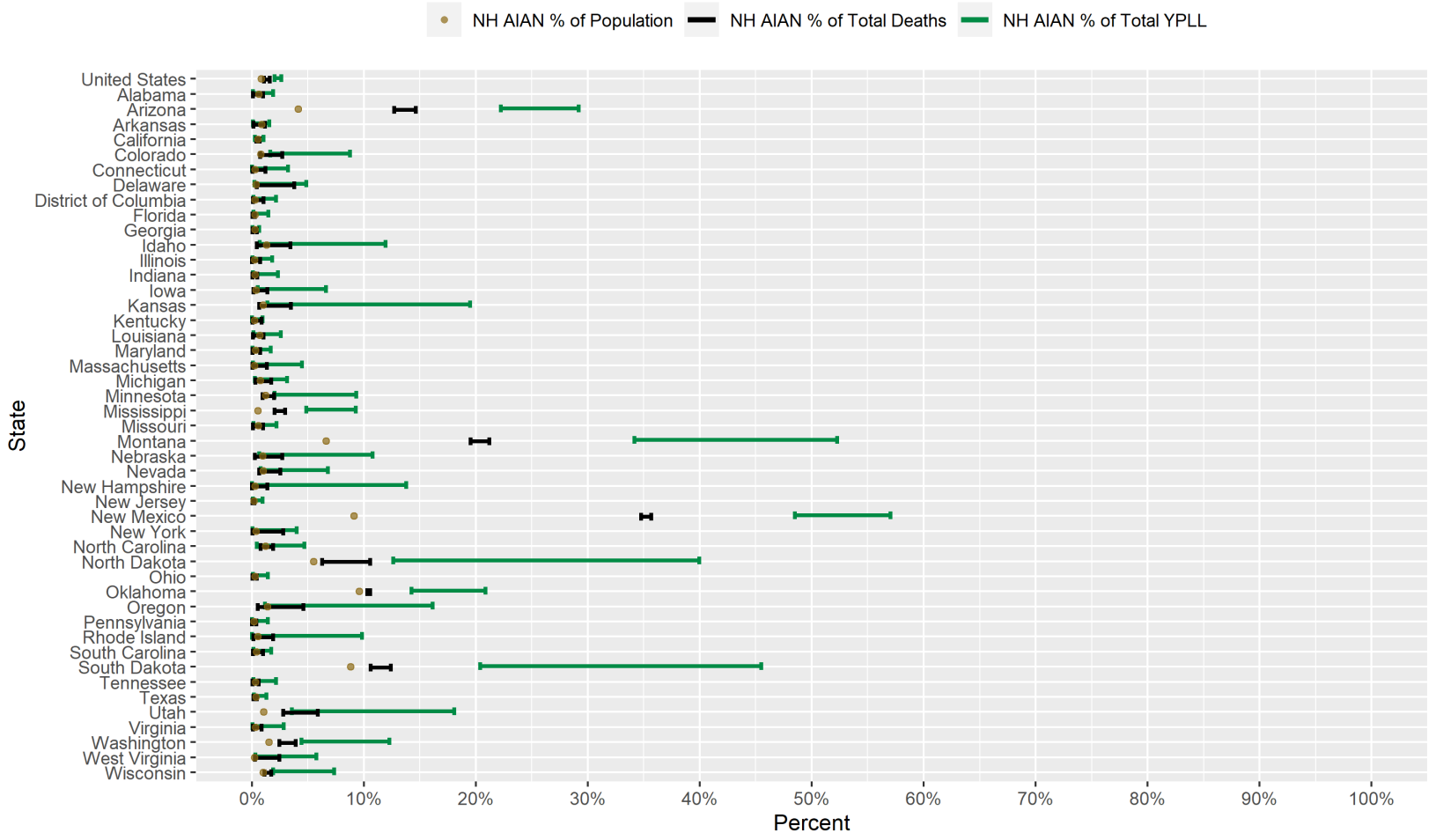

Figure 8. Conservative 95\% interval estimates of the percentage of total COVID-19-attributable YPLL before age 75, intervals denoting the entire plausible range for the percentage of total COVID-19 deaths, and the percent population shares for NH AIAN's in the U.S. and in each examined state with respect to cumulative COVID-19 deaths according to data from the National Center for Health Statistics as of 30 December 2020. 
Figure 9 presents a graphical comparison of the conservative 95\% interval estimates of the age-adjusted NH AIAN-to-NH White YPLL and mortality RR's in the U.S. and in each examined state. The U.S. national conservative 95\% interval estimate of the ageadjusted NH AIAN-to-NH White mortality RR is [1.82-2.70], and the state-level mortality RR interval estimates are completely above 1.0, 2.0, and 3.0 in 14, 8, and 6 of the examined states, respectfully. However, NH AIAN COVID-19 decedent ages in the U.S. overall are systematically younger relative to $\mathrm{NH}$ Whites, after accounting for differences in the national population age distributions between NH AIAN's and NH Whites, to such a degree that the U.S. national conservative $95 \%$ interval estimate of the age-adjusted $\mathrm{NH}$ AIAN-to-NH White YPLL RR is [3.79-5.06], completely above the U.S. national mortality RR interval estimate. At the state level, YPLL RR interval estimates are completely above the mortality RR interval estimates in 7 of the 46 examined states and completely below in none of the examined states, suggesting that the age-adjusted national trend of $\mathrm{NH}$ AIAN's dying from COVID-19 at younger ages relative to NH Whites is driven in large part by a small subset of states. Furthermore, state-level YPLL RR interval estimates are completely above 1.0, 2.0, and 3.0 in 20,14, and 11 of the examined states, respectfully, greater than the corresponding numbers for the state-level mortality RR interval estimates. The YPLL RR interval estimates in 4 of the examined states (which are among the 7 states where the YPLL RR interval estimates are completely above the mortality RR interval estimates) stand out starkly among the examined states with the highest age-adjusted $\mathrm{NH}$ AIAN-NH White disparities in COVID-19-attributable YPLL and are simply astronomical: Arizona ([14.79-23.70]), Mississippi ([17.97-34.13]), Montana ([11.14-22.46]), and New Mexico ([11.83-31.63]).

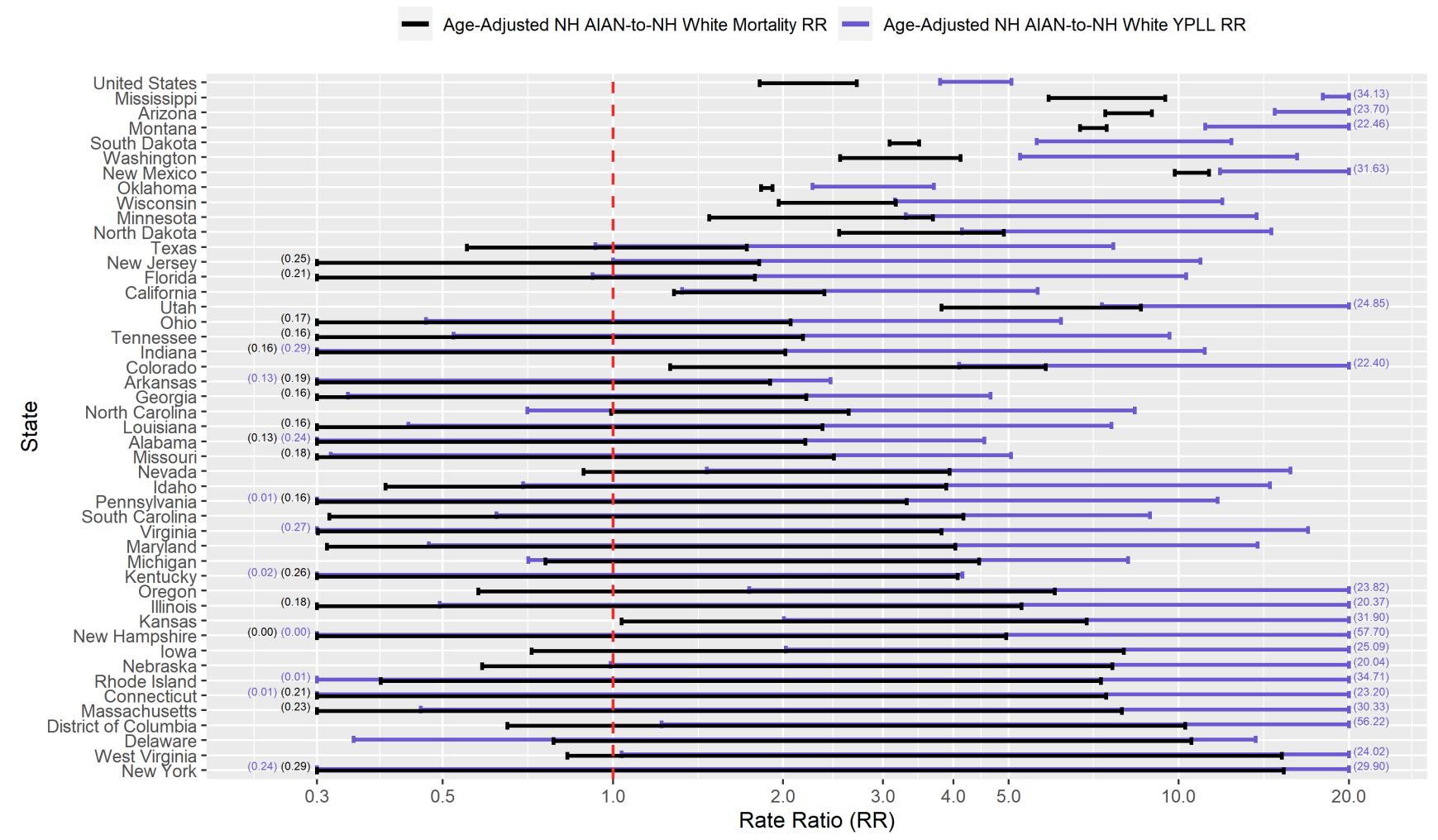

Figure 9. Conservative 95\% interval estimates of the age-adjusted NH AIAN-to-NH White YPLL and mortality RR's in the U.S. and in each examined state with respect to cumulative COVID-19 deaths according to data from the National Center for Health Statistics as of 30 December 2020. States are ordered from top to bottom in descending order of the signed difference between the lower limit of the YPLL RR interval and the upper limit of the mortality RR interval. Values are displayed on the base 10 logarithmic scale. Interval endpoints above 20.0 and below 0.30 are truncated, with the actual values numerically annotated. 


\section{Discussion}

Over 926,058 years of potential life before the age of 75 have been lost in the U.S. as a result of COVID-19, according to (lagged) NCHS data as of 30 December 2020, corresponding to 301,679 deaths. Proportionally scaling up this lower bound to the true number of COVID-19 deaths as of 30 December 2020 (342,577), as documented by the New York Times, results in an estimated lower bound of total U.S. COVID-19-attributable YPLL in excess of 1 million. However, the relative COVID-19 mortality burden by race/ethnicity has been far from equitable. Building upon the national comparative YPLL analysis by race/ethnicity by Bassett et al., we performed a corresponding state-by-state analysis, quantifying racial/ethnic disparities in COVID-19-attributable YPLL in 45 states and D.C. Because our YPLL analysis was performed at a more geographically precise level, it allowed for the identification of individual states where racial/ethnic disparities are particularly exacerbated and where younger non-NH White populations have been disproportionately devastated, as well as states that are exceptions to overarching national trends, which may aid in ascertaining important state-level characteristics that may be associated with racial/ethnic disparities in COVID-19-attributable YPLL.

Conventional analyses quantifying racial/ethnic disparities in COVID-19 mortality typically measure mortality in terms of death counts, calculating the percentage of total deaths by race/ethnicity, contrasting them with their respective percent population shares, and/or calculating (age-adjusted) mortality rates by race/ethnicity [32,33,72]. Measuring mortality in terms of YPLL, however, provides a complementary set of estimates that can capture disparities in both the number of deaths and the ages at death, which our results have shown to provide much added insights into the relative COVID-19 mortality burden by race/ethnicity. While substantial racial/ethnic disparities exist in COVID-19 mortality, they represent only one dimension of the disproportionate impact of COVID-19 on communities of color. For instance, substantial racial/ethnic disparities in COVID-19 cases also exist [11], and while the overwhelming majority of COVID-19 patients do not die from the disease, the long-term health effects post-infection are not even close to being fully understood at the time of writing [73]. When more detailed information on the long-term disability profiles of formerly COVID-19-infected individuals becomes available, a comparative analysis of disability-adjusted life years (DALY) [74] by race/ethnicity, for example, would augment our scientific understanding of the full extent of the disproportionate impacts of COVID-19 on communities of color.

Researchers have proposed multiple narratives to explain the vast disparities in the COVID-19 mortality burden by race/ethnicity. For example, Blacks and Hispanics in the U.S. have higher rates of underlying medical conditions such as diabetes, hypertension, and obesity [75,76], which have been established as definitive or probable risk factors of severe illness as a result of COVID-19 infection [77], and it has been hypothesized that the high prevalence of these conditions among Blacks and Hispanics in the U.S. contributes in large part to the observed disparities. Genetic factors have also been speculated as playing a role in the excess mortality experienced by Blacks in particular [78-80]. However, given that racial/ethnic disparities in the COVID-19 mortality burden reflect disparities in both the risk of COVID-19 infection and the risk of severe illness given COVID-19 infection, the underlying causes of the racial/ethnic disparities in the COVID-19 mortality burden are likely multi-faceted, comprising a complex and interactive combination of factors, including the prevalence of pre-existing comorbid conditions and potentially genetic factors. A consistent feature in the results of our analysis is the wide geographical variation in the magnitudes of the estimated racial/ethnic disparities in the COVID-19 mortality burden across states, clearly reflected in Figures 1-9. Some states have substantially higher magnitudes of racial/ethnic disparities than others, and even the direction of the disparity within a racial/ethnic group can differ state to state. This substantial state-to-state variation consequently suggests that racial/ethnic disparities in the COVID-19 mortality burden are driven in large part by social determinants of health [81] whose degree of association 
with race/ethnicity varies by state, a perspective widely propounded in the scientific community [82-87].

Social determinants of health that can shape different experiences of individuals in different racial/ethnic groups include health and health care, neighborhood and built environment, and economic stability [81]. Barriers to health care include lack of health insurance and health illiteracy. NH Black, Hispanic, and NH AIAN health insurance rates have historically lagged behind NH Whites [88,89]. A Gallup-West Health healthcare costs study conducted early in the U.S. COVID-19 epidemic (1-14 April 2020) found that $14 \%$ of non-White survey responds would avoid treatment for a suspected COVID-19 infection due to cost of care compared to only $6 \%$ of Whites [90]. NH Blacks, Hispanics, and NH AIAN's also suffer from disproportionately low rates of health literacy [91,92], which contributes to underutilization of preventive health services [93] and hinders the ability to process health information to make informative health decisions, a challenge especially exacerbated by the COVID-19 "infodemic" [94-100]. Poor housing conditions, which are inextricably associated with overcrowding and lack of sanitation, have been linked with increased rates of COVID-19 mortality [101], and NH Blacks and Hispanics are more likely to reside in moderate to severe substandard housing than NH Whites [102]. Blacks and Hispanics also generally experience higher rates of industrial air pollution [103], which may increase the risk of COVID-19 mortality [104,105]. Substantial income and wealth gaps exist across race/ethnicity [106], which contribute to racial/ethnic disparities in health care quality and subsequent health outcomes. Moreover, Blacks and Hispanics are overrepresented in many frontline essential industries [107-109] that require continued employment during the COVID-19 epidemic, resulting in higher rates of COVID-19 exposure and subsequent infection and mortality. Social determinants of health also include the domains of education and social and community context [81]; see Singu et al. (2020) [110] for a comprehensive treatment of social determinants of health in the context of COVID-19.

An important limitation of our analysis is that it is descriptive in nature (i.e., descriptive epidemiology [111]), primarily comprising the quantification of racial/ethnic disparities in COVID-19-attributable YPLL. Future work pinpointing exact reasons why certain states have particularly high or low disparities for certain racial/ethnic groups would vastly improve our understanding of the social conditions in which COVID-19 is most lethal as well as inform effective strategies for public health interventions. Despite this current gap in understanding, however, the sheer magnitude of many of the estimated state-level disparities in our analysis speaks to the degree of urgency for communities of color in relation to the U.S. COVID-19 epidemic.

At the time of writing, two COVID-19 vaccines, one manufactured by Pfizer, Inc. /BioNTech [112] and the other by ModernaTX, Inc. [113], have been granted emergency use authorization by the U.S. Food and Drug Administration, with several vaccine candidates in Phase III clinical trials. Vaccine distribution strategy has been the subject of rigorous public policy debate, with varying opinions on which segments of the population to prioritize for vaccination $[114,115]$. In any case, it is critical that communities of color particularly devastated by the U.S. COVID-19 epidemic have direct and equitable access to COVID-19 vaccines, but challenges already exist, especially with regards to vaccine hesitancy among Blacks. A Pew Research survey conducted 18-29 November 2020 [116] found that only $42 \%$ of $\mathrm{NH}$ Blacks would be willing to get a COVID-19 vaccine, well below the corresponding percentages for the other racial/ethnic groups considered in the survey, perhaps related to the well-documented mistrust by Blacks in the healthcare system and medical research [117-123]. A later national survey conducted 10-21 December 2020 by the National Foundation for Infectious Diseases [124] found that only $49 \%$ of Blacks planned to get a COVID-19 vaccine once available to them. The same survey also found that over half $(52 \%)$ of the survey respondents believed that the U.S. healthcare system "always/often" treats people unfairly based on their race or ethnic background. A recent study by Bogart et al. (2021) [125] also found substantial issues of mistrust surrounding COVID-19 vaccines and treatment among HIV-positive Blacks. Their study findings 
combined with experience and expertise regarding issues of medical mistrust by U.S. Blacks in the context of HIV [126,127] lead Bogart et al. to advocate for public health interventions encouraging COVID-19 vaccine uptake and treatment among Blacks to be grounded in community-based partnerships, tailoring community-specific strategies to overcome mistrust and access inequities. For example, offering COVID-19 vaccinations in nonmedical settings may increase vaccine uptake; partnering with faith-based organizations could help facilitate such efforts, a strategy that has already been employed in various local settings [128-133]. Nevertheless, while vaccination serves as the most potent tool to fight COVID-19, both vaccine-based and non-pharmaceutical interventions should be pursued to prevent the further devastation of communities of color from the U.S. COVID-19 epidemic and to confront future public health crises.

\section{Conclusions}

In summary, we performed an extensive secondary analysis of U.S. national COVID-19 death counts stratified by state, race/ethnicity, and age group from the National Center for Health Statistics as of 30 December 2020, quantifying racial/ethnic disparities in COVID-19attributable YPLL before age 75 in each of 45 states and D.C. Specifically, we quantified these disparities in YPLL through the estimation of percentages of total YPLL by race/ethnicity, contrasting them with their respective percent population shares, as well as age-adjusted YPLL RR's for NH Blacks, Hispanics, NH Asians, and NH AIAN's relative to NH Whites. Substantially complicating our analysis, however, were three sources of uncertainty in the data, namely, the administrative interval censoring of ages at death (precluding the exact calculation of YPLL), suppression of death counts between 1 and 9 within age intervals denoting age at death, and unknown race/ethnicity for a subset of COVID-19 deaths. We overcame these challenges in estimation by developing a novel adaptation of the MC simulation procedure proposed by $\mathrm{Xu}$ et al. (2021) that targets estimation of the extrema of the values of the estimands of interest that could theoretically be attained. However, a consequence of this conservative estimation strategy was wide interval estimates in scenarios for states and racial/ethnic groups corresponding to a high ratio of suppressed death counts to non-suppressed death counts and/or a comparatively large number of deaths with unknown race/ethnicity, which can reduce the power to detect racial/ethnic disparities when they exist and are small. Despite these challenges in estimation, our analysis revealed substantial racial/ethnic disparities in COVID-19-attributable YPLL before age 75 across U.S. states, with a prevailing pattern of NH Blacks and Hispanics experiencing disproportionately high COVID-19-attributable YPLL and NH Whites experiencing disproportionately low COVID-19-attributable YPLL. For comparison, we also calculated bounds for the corresponding percentages of total deaths and estimated the corresponding age-adjusted mortality RR's in the 45 examined states and D.C., which revealed that racial/ethnic disparities in the COVID-19 mortality burden are generally greater in magnitude when measuring mortality in terms of YPLL compared to (age-irrespective) death counts, reflecting the greater intensity of the disparities at younger ages. The substantial state-to-state variation in the magnitudes of the estimated racial/ethnic disparities suggests that these disparities are driven in large part by social determinants of health, whose degree of association with race/ethnicity varies by state. COVID-19 certainly didn't cause racial/ethnic disparities in health outcomes, but it did highlight and bring unprecedented national attention to long-standing societal and health inequalities that many communities of color in the U.S. face. It is imperative that we rise to the challenge of addressing the health needs of communities of color both during the U.S. COVID-19 epidemic and long after its conclusion.

Supplementary Materials: The following are available online at https://www.mdpi.com/1660 -4601/18/6/2921/s1, File S1: Cumulative COVID-19 death counts in the U.S. stratified by state, race/ethnicity, and age group from the National Center for Health Statistics as of 30 December 2020, File S2: Cumulative COVID-19 death counts in the U.S. stratified by state, sex, and age group from the National Center for Health Statistics as of 30 December 2020, File S3: 2019 CDC WONDER estimates 
of the U.S. population stratified by state, race, ethnicity, and age group, Table S1: Conservative 95\% interval estimates of total COVID-19-attributable YPLL before age 75 by race/ethnicity in the U.S. and in each examined state, Table S2: Intervals denoting the entire plausible range of total COVID-19 deaths by race/ethnicity in the U.S. and in each examined state, Table S3: Conservative 95\% interval estimates of the percentage of total COVID-19-attributable YPLL before age 75 and intervals denoting the entire plausible range of the percentage of total COVID-19 deaths by race/ethnicity in the U.S. and in each examined state, Table S4: Conservative 95\% interval estimates of age-adjusted YPLL and mortality rates by race/ethnicity in the U.S. and in each examined state, Table S5: Conservative 95\% interval estimates of the age-adjusted YPLL and mortality RR's for NH Blacks, Hispanics, NH Asians, and NH AIAN's relative to NH Whites in the U.S. and in each examined state.

Author Contributions: Conceptualization, J.J.X.; Data curation, J.J.X.; Formal analysis, J.J.X.; Methodology, J.J.X.; Software, J.J.X.; Visualization, J.J.X.; Writing—original draft, J.J.X.; Writing-review \& editing, J.J.X., J.T.C., T.R.B., R.S.B., M.A.S. and C.M.R. All authors have read and agreed to the published version of the manuscript.

Funding: This research received no external funding.

Institutional Review Board Statement: Not applicable.

Informed Consent Statement: Not applicable.

Data Availability Statement: The data presented in this study are available in the Supplementary Materials.

Conflicts of Interest: T.R.B. has received support from NIH/NCATS grant UL1 TR001881 and NIH/NIMH grant P30 MH058107 in addition to funding outside the scope of this work from the Patient Centered Outcomes Research Institute and the Movember Foundation. M.A.S. has received contracts from Janssen Research \& Development, LLC; Private Health Management, Inc.; the United States Department of Veteran Affairs; and the United States Food \& Drug Administration and research grants from the National Institutes of Health, all outside the scope of this work. C.M.R. has received a contract from Private Health Management, Inc. outside the scope of this work.

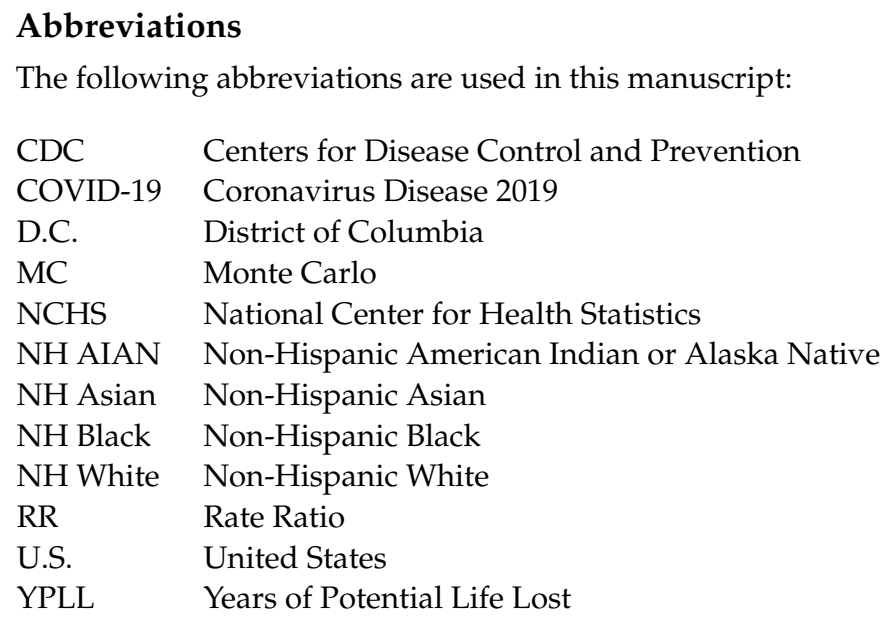

\section{References}

1. Cevik, M.; Kuppalli, K.; Kindrachuk, J.; Peiris, M. Virology, transmission, and pathogenesis of SARS-CoV-2. BMJ 2020, 371. [CrossRef] [PubMed]

2. Dong, E.; Du, H.; Gardner, L. An interactive web-based dashboard to track COVID-19 in real time. Lancet Infect. Dis. 2020, 20, 533-534. [CrossRef]

3. First Patient With Wuhan Coronavirus Is Identified in the U.S. Available online: https://www.nytimes.com/2020/01/21/health/ cdc-coronavirus.html (accessed on 15 February 2021).

4. Holshue, M.L.; DeBolt, C.; Lindquist, S.; Lofy, K.H.; Wiesman, J.; Bruce, H.; Spitters, C.; Ericson, K.; Wilkerson, S.; Tural, A.; et al. First Case of 2019 Novel Coronavirus in the United States. N. Engl. J. Med. 2020, 382, 929-936. [CrossRef] [PubMed]

5. Cucinotta, D.; Vanelli, M. WHO declares COVID-19 a pandemic. Acta Bio Med. 2020, 91, 157-160.

6. Coronavirus (Covid-19) Data in the United States. Available online: https://github.com/nytimes/covid-19-data (accessed on 15 February 2021). 
7. Griffith, D.M.; Sharma, G.; Holliday, C.S.; Enyia, O.K.; Valliere, M.; Semlow, A.R.; Stewart, E.C.; Blumenthal, R.S. Men and COVID-19: A Biopsychosocial Approach to Understanding Sex Differences in Mortality and Recommendations for Practice and Policy Interventions. Prev. Chronic Dis. 2020, 17, E63. [CrossRef]

8. Older Adults: At Greater Risk Of requiring Hospitalization or Dying if Diagnosed with COVID-19. Available online: https: //www.cdc.gov/coronavirus/2019-ncov/need-extra-precautions/older-adults.html (accessed on 15 February 2021).

9. Minority Groups at Risk as States withhold, Provide Partial COVID-19 Racial Data. Available online: https://abcnews.go.com/ Politics/states-missing-covid-19-racial-ethnic-data-creates/story?id=70338255 (accessed on 15 February 2021).

10. Krieger, N.; Gonsalves, G.; Bassett, M.; Hanage, W.; Krumholz, H. The Fierce Urgency Of Now: Closing Glaring Gaps In US Surveillance Data On COVID-19. Health Aff. Blog 2020. Available online: https://www.healthaffairs.org/do/10.1377/hblog202 00414.238084/full/ (accessed on 15 February 2021).

11. COVID-19 Hospitalization and Death by Race/Ethnicity. Available online: https://www.cdc.gov/coronavirus/2019-ncov/ covid-data/investigations-discovery/hospitalization-death-by-race-ethnicity.html (accessed on 15 February 2021).

12. Millett, G.A.; Jones, A.T.; Benkeser, D.; Baral, S.; Mercer, L.; Beyrer, C.; Honermann, B.; Lankiewicz, E.; Mena, L.; Crowley, J.S.; et al. Assessing differential impacts of COVID-19 on Black communities. Ann. Epidemiol. 2020, 47, 37-44. [CrossRef]

13. Karaca-Mandic, P.; Georgiou, A.; Sen, S. Assessment of COVID-19 Hospitalizations by Race/Ethnicity in 12 States. JAMA Intern. Med. 2021, 181, 131-134. [CrossRef]

14. Holtgrave, D.R.; Barranco, M.A.; Tesoriero, J.M.; Blog, D.S.; Rosenberg, E.S. Assessing racial and ethnic disparities using a COVID-19 outcomes continuum for New York State. Ann. Epidemiol. 2020, 48, 9-14. [CrossRef]

15. Golestaneh, L.; Neugarten, J.; Fisher, M.; Billett, H.H.; Gil, M.R.; Johns, T.; Yunes, M.; Mokrzycki, M.H.; Coco, M.; Norris, K.C.; et al. The association of race and COVID-19 mortality. EClinicalMedicine 2020, 25, 100455. [CrossRef]

16. Renelus, B.D.; Khoury, N.C.; Chandrasekaran, K.; Bekele, E.; Briggs, W.M.; Ivanov, A.; Mohanty, S.R.; Jamorabo, D.S. Racial Disparities in COVID-19 Hospitalization and In-hospital Mortality at the Height of the New York City Pandemic. J. Racial Ethn. Health Disparities 2020. [CrossRef]

17. Lieberman-Cribbin, W.; Tuminello, S.; Flores, R.M.; Taioli, E. Disparities in COVID-19 Testing and Positivity in New York City. Am. J. Prev. Med. 2020, 59, 326-332. [CrossRef] [PubMed]

18. Rodriguez-Diaz, C.E.; Guilamo-Ramos, V.; Mena, L.; Hall, E.; Honermann, B.; Crowley, J.S.; Baral, S.; Prado, G.J.; MarzanRodriguez, M.; Beyrer, C.; et al. Risk for COVID-19 infection and death among Latinos in the United States: Examining heterogeneity in transmission dynamics. Ann. Epidemiol. 2020, 52, 46-53. [CrossRef] [PubMed]

19. Gross, C.P.; Essien, U.R.; Pasha, S.; Gross, J.R.; Wang, S.y.; Nunez-Smith, M. Racial and Ethnic Disparities in Population-Level Covid-19 Mortality. J. Gen. Intern. Med. 2020, 35, 3097-3099. [CrossRef] [PubMed]

20. Arasteh, K. Prevalence of Comorbidities and Risks Associated with COVID-19 Among Black and Hispanic Populations in New York City: An Examination of the 2018 New York City Community Health Survey. J. Racial Ethn. Health Disparities 2020. [CrossRef]

21. Abedi, V.; Olulana, O.; Avula, V.; Chaudhary, D.; Khan, A.; Shahjouei, S.; Li, J.; Zand, R. Racial, Economic, and Health Inequality and COVID-19 Infection in the United States. J. Racial Ethn. Health Disparities 2020. [CrossRef]

22. Thomas, M.D.; Michaels, E.K.; Darling-Hammond, S.; Nguyen, T.T.; Glymour, M.M.; Vittinghoff, E. Whites' County-Level Racial Bias, COVID-19 Rates, and Racial Inequities in the United States. Int. J. Environ. Res. Public Health 2020, 17, 8695. [CrossRef]

23. Zelner, J.; Trangucci, R.; Naraharisetti, R.; Cao, A.; Malosh, R.; Broen, K.; Masters, N.; Delamater, P. Racial Disparities in Coronavirus Disease 2019 (COVID-19) Mortality Are Driven by Unequal Infection Risks. Clin. Infect. Dis. 2020, 72, e88-e95. [CrossRef]

24. Wang, Q.; Berger, N.A.; Xu, R. Analyses of Risk, Racial Disparity, and Outcomes Among US Patients With Cancer and COVID-19 Infection. JAMA Oncol. 2020, 7, 220-227. [CrossRef]

25. Tirupathi, R.; Muradova, V.; Shekhar, R.; Salim, S.A.; Al-Tawfiq, J.A.; Palabindala, V. COVID-19 disparity among racial and ethnic minorities in the US: A cross sectional analysis. Travel Med. Infect. Dis. 2020, 38, 101904. [CrossRef]

26. Muñoz-Price, L.S.; Nattinger, A.B.; Rivera, F.; Hanson, R.; Gmehlin, C.G.; Perez, A.; Singh, S.; Buchan, B.W.; Ledeboer, N.A.; Pezzin, L.E. Racial Disparities in Incidence and Outcomes Among Patients With COVID-19. JAMA Netw. Open 2020, 3, e2021892-e2021892. [CrossRef]

27. Parcha, V.; Malla, G.; Suri, S.S.; Kalra, R.; Heindl, B.; Berra, L.; Fouad, M.N.; Arora, G.; Arora, P. Geographic Variation in Racial Disparities in Health and Coronavirus Disease-2019 (COVID-19) Mortality. Mayo Clin. Proc. Innov. Qual. Outcomes 2020, 4, 703-716. [CrossRef]

28. Gu, T.; Mack, J.A.; Salvatore, M.; Sankar, S.P.; Valley, T.S.; Singh, K.; Nallamothu, B.K.; Kheterpal, S.; Lisabeth, L.; Fritsche, L.G.; et al. Characteristics Associated With Racial/Ethnic Disparities in COVID-19 Outcomes in an Academic Health Care System. JAMA Netw. Open 2020, 3, e2025197. [CrossRef]

29. Vahidy, F.S.; Nicolas, J.C.; Meeks, J.R.; Khan, O.; Pan, A.; Jones, S.L.; Masud, F.; Sostman, H.D.; Phillips, R.; Andrieni, J.D.; et al. Racial and ethnic disparities in SARS-CoV-2 pandemic: analysis of a COVID-19 observational registry for a diverse US metropolitan population. BMJ Open 2020, 10, e039849. [CrossRef] [PubMed]

30. Ogedegbe, G.; Ravenell, J.; Adhikari, S.; Butler, M.; Cook, T.; Francois, F.; Iturrate, E.; Jean-Louis, G.; Jones, S.A.; Onakomaiya, D.; et al. Assessment of Racial/Ethnic Disparities in Hospitalization and Mortality in Patients With COVID-19 in New York City. JAMA Netw. Open 2020, 3, e2026881. [CrossRef] [PubMed] 
31. Strully, K.; Yang, T.C.; Liu, H. Regional variation in COVID-19 disparities: connections with immigrant and Latinx communities in US counties. Ann. Epidemiol. 2021, 53, 56-62. [CrossRef] [PubMed]

32. The Color of Coronavirus: COVID-19 Deaths by Race and Ethnicity in the U.S. Available online: https://www.apmresearchlab. org/covid/deaths-by-race (accessed on 15 February 2021).

33. The COVID Racial Data Tracker. Available online: https:/ / covidtracking.com/race (accessed on 15 February 2021).

34. Gardner, J.W.; Sanborn, J.S. Years of Potential Life Lost (YPLL)—What Does it Measure? Epidemiology 1990, 1, 322-329. [CrossRef] [PubMed]

35. Desenclos, J.C.A.; Hahn, R.A. Years of potential life lost before age 65, by race, Hispanic origin, and sex-United States, $1986-1988$. Morb. Mortal. Wkly. Rep. CDC Surveill. Summ. 1992, 41, 13-23.

36. Li, C.; Ekwueme, D.U.; Rim, S.H.; Tangka, F.K. Years of Potential Life Lost and Productivity Losses From Male Urogenital Cancer Deaths-United States, 2004. Urology 2010, 76, 528-535. [CrossRef]

37. Max, W.; Sung, H.Y.; Shi, Y. Deaths From Secondhand Smoke Exposure in the United States: Economic Implications. Am. J. Public Health 2012, 102, 2173-2180. [CrossRef] [PubMed]

38. Bassett, M.T.; Chen, J.T.; Krieger, N. Variation in racial/ethnic disparities in COVID-19 mortality by age in the United States: A cross-sectional study. PLoS Med. 2020, 17, e1003402. [CrossRef] [PubMed]

39. Deaths Involving Coronavirus Disease 2019 (COVID-19) by Race and Hispanic Origin Group and Age, by State. Available online: https: / / data.cdc.gov/NCHS/Deaths-involving-coronavirus-disease-2019-COVID-19/ks3g-spdg (accessed on 15 February 2021).

40. U.S. Deaths Are Up Sharply, Though Covid-19's Precise Toll Is Murky. Available online: https://www.wsj.com/articles/covid19s-exact-toll-is-murky-though-u-s-deaths-are-up-sharply-11589555652 (accessed on 15 February 2021).

41. Excess Deaths From COVID-19 and Other Causes, March-April 2020, author=Woolf, Steven H and Chapman, Derek A and Sabo, Roy $\mathrm{T}$ and Weinberger, Daniel $\mathrm{M}$ and Hill, Latoya. JAMA 2020, 324, 510-513. [CrossRef] [PubMed]

42. Weinberger, D.M.; Chen, J.; Cohen, T.; Crawford, F.W.; Mostashari, F.; Olson, D.; Pitzer, V.E.; Reich, N.G.; Russi, M.; Simonsen, L.; et al. Estimation of Excess Deaths Associated With the COVID-19 Pandemic in the United States, March to May 2020. JAMA Intern. Med. 2020, 180, 1336-1344. [CrossRef] [PubMed]

43. Why is NC Delayed in Reporting COVID-19 Death Data? It's About Death Certificates. Available online: https://www northcarolinahealthnews.org/2020/09/18/why-is-nc-delayed-in-reporting-covid-19-death-data/ (accessed on 15 February 2021).

44. Provisional COVID-19 Death Counts by Sex, Age, and State. Available online: https:/ / data.cdc.gov/NCHS/Provisional-COVID19-Death-Counts-by-Sex-Age-and-S/9bhg-hcku (accessed on 15 February 2021).

45. Bridged-Race Population Estimates 1990-2019 Request. Available online: https://wonder.cdc.gov/Bridged-Race-v2019.HTML (accessed on 30 December 2020).

46. Rothman, K.J.; Greenland, S.; Lash, T.L. Modern Epidemiology; Lippincott Williams \& Wilkins: Philadelphia, PA, USA, 2008.

47. Romeder, J.; McWhinnie, J. Potential Years of Life Lost Between Ages 1 and 70: An Indicator of Premature Mortality for Health Planning. Int. J. Epidemiol. 1977, 6, 143-151. [CrossRef] [PubMed]

48. Arcà, M.; Di Orio, F.; Forastiere, F.; Tasco, C.; Perucci, C. Years of Potential Life Lost (YPLL) before Age 65 in Italy. Am. J. Public Health 1988, 78, 1202-1205. [CrossRef]

49. Curran, J.W.; Morgan, W.M.; Hardy, A.M.; Jaffe, H.W.; Darrow, W.W.; Dowdle, W.R. The epidemiology of AIDS: Current status and future prospects. Science 1985, 229, 1352-1357. [CrossRef]

50. Albert, V.A.; Koh, H.K.; Geller, A.C.; Miller, D.R.; Prout, M.N.; Lew, R.A. Years of potential life lost: Another indicator of the impact of cutaneous malignant melanoma on society. J. Am. Acad. Dermatol. 1990, 23, 308-310. [CrossRef]

51. Mitra, A.K.; Payton, M.; Kabir, N.; Whitehead, A.; Ragland, K.N.; Brown, A. Potential Years of Life Lost Due to COVID-19 in the United States, Italy, and Germany: An Old Formula with Newer Ideas. Int. J. Environ. Res. Public Health 2020, 17, 4392. [CrossRef]

52. Xu, J.J.; Belin, T.R.; Ramirez, C.M. Uncertainty Quantification of Years of Potential Life Lost-Based Estimates from Mortality Data Summarized as Death Counts Within Age Intervals. Ann. Epidemiol. 2021, 55, 1-3. [CrossRef]

53. Bobashev, G.V.; Morris, R.J. Uncertainty and Inference in Agent-based Models. In Proceedings of the 2010 Second International Conference on Advances in System Simulation, Nice, France, 22-27 August 2010; pp. 67-71.

54. Celentano, D.D.; Szklo, M. Gordis Epidemiology; Elsevier: Philadelphia, PA, USA, 2019.

55. Racial Data Transparency. Available online: https://coronavirus.jhu.edu/data/racial-data-transparency (accessed on 15 February 2021).

56. Table 25. Years of Potential Life Lost before Age 75 for Selected Causes of Death, by Sex, Race, and Hispanic Origin: United States, Selected Years 1980-2007. Available online: https://www.cdc.gov/nchs/data/hus/2010/025.pdf (accessed on 15 February 2021).

57. Table 25. Years of Potential Life Lost before age 75 for Selected Causes of Death, by Sex, Race, and Hispanic Origin: United States, Selected Years 1980-2010. Available online: https://www.cdc.gov/nchs/data/hus/2011/025.pdf (accessed on 15 February 2021).

58. Table 21. Years of Potential life Lost before Age 75 for Selected Causes of Death, by Sex, Race, and Hispanic Origin: United States, Selected Years 1980-2010. Available online: https:/ /www.cdc.gov/nchs/data/hus/2012/021.pdf (accessed on 15 February 2021).

59. Table 21. Years of Potential Life lost Before age 75 For selected Causes of Death, by Sex, Race, and Hispanic Origin: United States, Selected Years 1980-2011. Available online: https://www.cdc.gov/nchs/data/hus/2013/021.pdf (accessed on 15 February 2021).

60. Table 19. Years of Potential Life Lost before Age 75 for Selected Causes of Death, by Sex, Race, and Hispanic Origin: United States, Selected Years 1980-2013. Available online: https:/ /www.cdc.gov/nchs/data/hus/2014/019.pdf (accessed on 15 February 2021). 
61. Table 18 (page 1 of 4). Years of Potential Life Lost before Age 75 for Selected Causes of Death, by Sex, Race, and Hispanic Origin: United States, Selected Years 1980-2014. Available online: https:/ /www.cdc.gov/nchs/data/hus/2015/018.pdf (accessed on 15 February 2021).

62. Table 18. Years of Potential Life Lost before Age 75 for Selected Causes of Death, by Sex, Race, and Hispanic Origin: United States, Selected Years 1980-2015. Available online: https:/ /www.cdc.gov/nchs/data/hus/2016/018.pdf (accessed on 15 February 2021).

63. Table 18. Years of Potential Life Lost before age 75 for Selected Causes of Death, by Sex, Race, and Hispanic Origin: United States, Selected Years 1980-2016. Available online: https://www.cdc.gov/nchs/data/hus/2017/018.pdf (accessed on 15 February 2021).

64. Borse, N.N.; Rudd, R.A.; Dellinger, A.M.; Sleet, D.A. Years of potential life lost from unintentional child and adolescent injuries-United States, 2000-2009. J. Saf. Res. 2013, 45, 127-131. [CrossRef]

65. Khalil, M.I.; Bimali, M.; Davis, R.; Machado, B.; Kamel, M. MP23-06 Racial Disparities in Years of Potential Life Lost Secondary to Untreated Low and Intermediate Risk Prostate Cancer Deaths. J. Urol. 2020, 203, e339-e340. [CrossRef]

66. Ekwueme, D.U.; Guy, G.P. Jr.; Li, C.; Rim, S.H.; Parelkar, P.; Chen, S.C. The health burden and economic costs of cutaneous melanoma mortality by race/ethnicity-United States, 2000 to 2006. J. Am. Acad. Dermatol. 2011, 65, S133..e1-S133.e12. [CrossRef]

67. Ekwueme, D.U.; Guy, G.P. Jr.; Rim, S.H.; White, A.; Hall, I.J.; Fairley, T.L.; Dean, H.D. Health and Economic Impact of Breast Cancer Mortality in Young Women, 1970-2008. Am. J. Prev. Med. 2014, 46, 71-79. [CrossRef] [PubMed]

68. Bucholz, E.M.; Normand, S.L.T.; Wang, Y.; Ma, S.; Lin, H.; Krumholz, H.M. Life Expectancy and Years of Potential Life Lost After Acute Myocardial Infarction by Sex and Race A Cohort-Based Study of Medicare Beneficiaries. J. Am. Coll. Cardiol. 2015, 66, 645-655. [CrossRef]

69. Iyer, D.G.; Shah, N.S.; Hastings, K.G.; Hu, J.; Rodriguez, F.; Boothroyd, D.B.; Krishnan, A.V.; Falasinnu, T.; Palaniappan, L. Years of Potential Life Lost Because of Cardiovascular Disease in Asian-American Subgroups, 2003-2012. J. Am. Heart Assoc. 2019, 8, e010744. [CrossRef] [PubMed]

70. Buchanich, J.M.; Doerfler, S.M.; Lann, M.F.; Marsh, G.M.; Burke, D.S. Improvement in racial disparities in years of life lost in the USA since 1990. PLoS ONE 2018, 13, e0194308. [CrossRef] [PubMed]

71. R Core Team. R: A Language and Environment for Statistical Computing; R Foundation for Statistical Computing: Vienna, Austria, 2019.

72. COVID-19 Deaths by Race/Ethnicity. Available online: https://www.kff.org/other/state-indicator/covid-19-deaths-by-raceethnicity/ (accessed on 15 February 2021).

73. Long-Term Effects of COVID-19. Available online: https://www.cdc.gov/coronavirus/2019-ncov/long-term-effects.html (accessed on 15 February 2021).

74. Murray, C.J. Quantifying the burden of disease: the technical basis for disability-adjusted life years. Bull. World Health Organ. 1994, 72, 429-445.

75. Profile: Black/African Americans. Available online: https://minorityhealth.hhs.gov/omh/browse.aspx?lvl=3\&lvlid=61 (accessed on 15 February 2021).

76. Profile: Hispanic/Latino Americans. Available online: https://minorityhealth.hhs.gov/omh/browse.aspx?lvl=3\&lvlid=64 (accessed on 15 February 2021).

77. People with Certain Medical Conditions. Available online: https://www.cdc.gov/coronavirus/2019-ncov/need-extraprecautions / people-with-medical-conditions.html (accessed on 15 February 2021).

78. Giudicessi, J.R.; Roden, D.M.; Wilde, A.A.; Ackerman, M.J. Genetic susceptibility for COVID-19-associated sudden cardiac death in African Americans. Heart Rhythm 2020, 17, 1487-1492. [CrossRef]

79. Bunyavanich, S.; Grant, C.; Vicencio, A. Racial/Ethnic Variation in Nasal Gene Expression of Transmembrane Serine Protease 2 (TMPRSS2). JAMA 2020, 324, 1567-1568. [CrossRef] [PubMed]

80. Sarangarajan, R.; Winn, R.; Kiebish, M.A.; Bountra, C.; Granger, E.; Narain, N.R. Ethnic Prevalence of AngiotensinConverting Enzyme Deletion (D) Polymorphism and COVID-19 Risk: Rationale for Use of Angiotensin-Converting Enzyme Inhibitors/Angiotensin Receptor Blockers. J. Racial Ethn. Health Disparities 2020. [CrossRef]

81. Social Determinants of Health. Available online: https://health.gov/healthypeople/objectives-and-data/social-determinantshealth (accessed on 15 February 2021).

82. Abrams, E.M.; Szefler, S.J. COVID-19 and the impact of social determinants of health. Lancet. Respir. Med. 2020, 8, 659-661. [CrossRef]

83. Shah, G.H.; Shankar, P.; Schwind, J.S.; Sittaramane, V. The Detrimental Impact of the COVID-19 Crisis on Health Equity and Social Determinants of Health. J. Public Health Manag. Pract. 2020, 26, 317-319. [CrossRef] [PubMed]

84. Rollston, R.; Galea, S. COVID-19 and the Social Determinants of Health. Am. J. Health Promot. 2020, 34, 687-689. [CrossRef] [PubMed]

85. Hooper, M.W.; Nápoles, A.M.; Pérez-Stable, E.J. COVID-19 and Racial/Ethnic Disparities. JAMA 2020, 323, 2466-2467. [CrossRef] [PubMed]

86. Maness, S.B.; Merrell, L.; Thompson, E.L.; Griner, S.B.; Kline, N.; Wheldon, C. Social Determinants of Health and Health Disparities: COVID-19 Exposures and Mortality Among African American People in the United States. Public Health Rep. 2021, 136, 18-22. [CrossRef] [PubMed]

87. Dalsania, A.K.; Fastiggi, M.J.; Kahlam, A.; Shah, R.; Patel, K.; Shiau, S.; Rokicki, S.; DallaPiazza, M. The Relationship Between Social Determinants of Health and Racial Disparities in COVID-19 Mortality. J. Racial Ethn. Health Disparities 2021. [CrossRef] 
88. Changes in Health Coverage by Race and Ethnicity since the ACA, 2010-2018. Available online: https://www.kff.org/racialequity-and-health-policy/issue-brief/changes-in-health-coverage-by-race-and-ethnicity-since-the-aca-2010-2018/ (accessed on 15 February 2021).

89. U.S. Uninsured Rate Steady at $12.2 \%$ in Fourth Quarter of 2017. Available online: https://news.gallup.com/poll/225383 / uninsured-rate-steady-fourth-quarter-2017.aspx (accessed on 15 February 2021).

90. In U.S., 14\% With Likely COVID-19 to Avoid Care Due to Cost. Available online: https://news.gallup.com/poll/309224/avoidcare-likely-covid-due-cost.aspx (accessed on 15 February 2021).

91. Kutner, M.; Greenburg, E.; Jin, Y.; Paulsen, C. The Health Literacy of America's Adults: Results from the 2003 National Assessment of Adult Literacy (NCES 2006-483). Natl. Cent. Educ. Stat. 2006.

92. Cutilli, C.C.; Bennett, I.M. Understanding the Health Literacy of America Results of the National Assessment of Adult Literacy. Orthop. Nurs. 2009, 28, 27-34. [CrossRef]

93. Benjamin, R.M. Improving Health by Improving Health Literacy. Public Health Rep. 2010, 125, 784-785. [CrossRef]

94. Zarocostas, J. How to fight an infodemic. Lancet 2020, 395, 676. [CrossRef]

95. Managing the COVID-19 Infodemic: Promoting Healthy Behaviours and Mitigating the Harm from Misinformation and Disinformation. Available online: https:/ / www.who.int/news/item/23-09-2020-managing-the-covid-19-infodemic-promotinghealthy-behaviours-and-mitigating-the-harm-from-misinformation-and-disinformation (accessed on 15 February 2021).

96. Diseases, T.L.I. The COVID-19 infodemic. Lancet Infect. Dis. 2020, 20, 875. [CrossRef]

97. Orso, D.; Federici, N.; Copetti, R.; Vetrugno, L.; Bove, T. Infodemic and the spread of fake news in the COVID-19-era. Eur. J. Emerg. Med. 2020, 27, 327-328. [CrossRef] [PubMed]

98. Cinelli, M.; Quattrociocchi, W.; Galeazzi, A.; Valensise, C.M.; Brugnoli, E.; Schmidt, A.L.; Zola, P.; Zollo, F.; Scala, A. The COVID-19 social media infodemic. Sci. Rep. 2020, 10,1-10. [CrossRef]

99. Siebenhaar, K.U.; Köther, A.K.; Alpers, G.W. Dealing With the COVID-19 Infodemic: Distress by Information, Information Avoidance, and Compliance With Preventive Measures. Front. Psychol. 2020, 11, 567905. [CrossRef]

100. Naeem, S.B.; Bhatti, R. The Covid-19 'infodemic': A new front for information professionals. Health Inf. Libr. J. 2020, 37, 233-239. [CrossRef]

101. Ahmad, K.; Erqou, S.; Shah, N.; Nazir, U.; Morrison, A.R.; Choudhary, G.; Wu, W.C. Association of poor housing conditions with COVID-19 incidence and mortality across US counties. PLoS ONE 2020, 15, e241327. [CrossRef] [PubMed]

102. Jacobs, D.E. Environmental Health Disparities in Housing. Am. J. Public Health 2011, 101, S115-S122. [CrossRef]

103. Zwickl, K.; Ash, M.; Boyce, J.K. Regional variation in environmental inequality: Industrial air toxics exposure in US cities. Ecol. Econ. 2014, 107, 494-509. [CrossRef]

104. Wu, X.; Nethery, R.; Sabath, M.; Braun, D.; Dominici, F. Air pollution and COVID-19 mortality in the United States: Strengths and limitations of an ecological regression analysis. Sci. Adv. 2020, 6, eabd4049. [CrossRef]

105. Pozzer, A.; Dominici, F.; Haines, A.; Witt, C.; Münzel, T.; Lelieveld, J. Regional and global contributions of air pollution to risk of death from COVID-19. Cardiovasc. Res. 2020, 116, 2247-2253. [CrossRef] [PubMed]

106. Williams, D.R.; Priest, N.; Anderson, N.B. Understanding Associations between Race, Socioeconomic Status and Health: Patterns and Prospects. Health Psychol. 2016, 35, 407-411. [CrossRef]

107. Labor Force Statistics from the Current Population Survey. Household Data. Annual Averages. 18. Employed Persons by Detailed Industry, Sex, Race, and Hispanic or Latino Ethnicity. Available online: https:/ /www.bls.gov/cps/cpsaat18.htm (accessed on 15 February 2021).

108. Rogers, T.N.; Rogers, C.R.; VanSant-Webb, E.; Gu, L.Y.; Yan, B.; Qeadan, F. Racial Disparities in COVID-19 Mortality Among Essential Workers in the United States. World Med. Health Policy 2020, 12, 311-327. [CrossRef] [PubMed]

109. Selden, T.M.; Berdahl, T.A. COVID-19 And Racial/Ethnic Disparities In Health Risk, Employment, And Household Composition. Health Aff. 2020, 39, 1624-1632. [CrossRef]

110. Singu, S.; Acharya, A.; Challagundla, K.; Byrareddy, S.N. Impact of Social Determinants of Health on the Emerging COVID-19 Pandemic in the United States. Front. Public Health 2020, 8. [CrossRef] [PubMed]

111. Porta, M. A Dictionary of Epidemiology; Oxford University Press: Oxford, UK, 2014.

112. Information about the Pfizer-BioNTech COVID-19 Vaccine. Available online: https:/ /www.cdc.gov/coronavirus/2019-ncov/ vaccines / different-vaccines/Pfizer-BioNTech.html (accessed on 15 February 2021).

113. Information about the Moderna COVID-19 Vaccine. Available online: https://www.cdc.gov/coronavirus/2019-ncov/vaccines/ different-vaccines/Moderna.html (accessed on 15 February 2021).

114. People Are Dying. Whom Do We Save First With the Vaccine? Available online: https://www.nytimes.com/2020/12/24 / magazine/who-should-get-the-covid-vaccine-next.html (accessed on 15 February 2021).

115. The Elderly vs. Essential Workers: Who Should Get the Coronavirus Vaccine First? Available online: https://www.nytimes.com/ 2020/12/05/health/covid-vaccine-first.html (accessed on 15 February 2021).

116. Funk, C.; Tyson, A. Intent to Get a COVID-19 Vaccine Rises to 60\% as Confidence in Research and Development Process Increases. Pew Res. Cent. 2020.

117. LaVeist, T.A.; Nickerson, K.J.; Bowie, J.V. Attitudes about Racism, Medical Mistrust, and Satisfaction with Care among African American and White Cardiac Patients. Med Care Res. Rev. 2000, 57, 146-161. [CrossRef] 
118. Corbie-Smith, G.; Thomas, S.B.; George, D.M.M.S. Distrust, Race, and Research. Arch. Intern. Med. 2002, 162, $2458-2463$. [CrossRef]

119. Boulware, L.E.; Cooper, L.A.; Ratner, L.E.; LaVeist, T.A.; Powe, N.R. Race and Trust in the Health Care System. Public Health Reports Jul-Aug 2003 2003, 118, 358-365. [CrossRef]

120. Halbert, C.H.; Armstrong, K.; Gandy, O.H.; Shaker, L. Racial Differences in Trust in Health Care Providers. Arch. Intern. Med. 2006, 166, 896-901. [CrossRef] [PubMed]

121. Kennedy, B.R.; Mathis, C.C.; Woods, A.K. African Americans and Their Distrust of the Health Care System: Healthcare for Diverse Populations. J. Cult. Divers. 2007, 14, 56-60. [PubMed]

122. Armstrong, K.; McMurphy, S.; Dean, L.T.; Micco, E.; Putt, M.; Halbert, C.H.; Schwartz, J.S.; Sankar, P.; Pyeritz, R.E.; Bernhardt, B.; et al. Differences in the Patterns of Health Care System Distrust Between Blacks and Whites. J. Gen. Intern. Med. 2008, 23, 827-833. [CrossRef]

123. Musa, D.; Schulz, R.; Harris, R.; Silverman, M.; Thomas, S.B. Trust in the Health Care System and the Use of Preventive Health Services by Older Black and White Adults. Am. J. Public Health 2009, 99, 1293-1299. [CrossRef] [PubMed]

124. National Survey: Black Adult Perspectives on COVID-19 and Flu Vaccines. Available online: https://www.nfid.org/nationalsurvey-black-adult-perspectives-on-covid-19-and-flu-vaccines / (accessed on 15 February 2021).

125. Bogart, L.M.; Ojikutu, B.O.; Tyagi, K.; Klein, D.J.; Mutchler, M.G.; Dong, L.; Lawrence, S.J.; Thomas, D.R.; Kellman, S. COVID-19 Related Medical Mistrust, Health Impacts, and Potential Vaccine Hesitancy Among Black Americans Living With HIV. J. Acquir. Immune Defic. Syndr. 2021, 86, 200-207. [CrossRef] [PubMed]

126. Bogart, L.M.; Takada, S.; Cunningham, W.E. Medical Mistrust, Discrimination, and the Domestic HIV Epidemic. In HIV in US Communities of Color; Springer Nature Switzerland AG: Cham, Switzerland, 2021; pp. 207-231.

127. Medical Mistrust Could Reduce Vaccine Uptake: Q\&A with Laura Bogart. Available online: https://www.rand.org/blog/randreview /2020/12/medical-mistrust-could-reduce-vaccine-uptake-qa-with.html (accessed on 15 February 2021).

128. Churches Pair Up with Clinics to Deliver Coronavirus Vaccine to Those Who Need It Most. Available online: https: / / www.washingtonpost.com/local/churches-coronavirus-vaccine-clinics/2021/02/10/522025f8-6b17-11eb-ba56-d7e2c8 defa31_story.html (accessed on 15 February 2021).

129. COVID-19 Vaccine: Reaching Black Ohioans with Shots in a Place they Trust-The Church. Available online: https://www. cincinnati.com/story/news/2021/02/10/covid-19-vaccine-black-church-cincinnati/4455725001/ (accessed on 15 February 2021).

130. Historic Black Denver Church Opens Doors For COVID Vaccination Event. Available online: https://denver.cbslocal.com/2021 /02/07/black-denver-church-covid-vaccination-event-shorter-community-ame/ (accessed on 15 February 2021).

131. Jackson is Partnering with Houses of Worship to Vaccinate Members. Is yours on the list? Available online: https://www. miamiherald.com/news/coronavirus/article248477485.html (accessed on 15 February 2021).

132. Novant and Churches Partner to help Distribute COVID-19 Vaccine. Available online: https://myfox8.com/news/coronavirus/ novant-and-churches-partner-to-help-distribute-covid-19-vaccine/ (accessed on 15 February 2021).

133. St. Dominic Hospital Teams Up with Area Churches to Provide COVID vaccine. Available online: https://www.wlbt.com/2021 /02/11/st-dominic-hospital-teams-up-with-area-churches-provide-covid-vaccine/ (accessed on 15 February 2021). 